\title{
Systematic Analysis of HD-ZIP Transcription Factors in Sesame Genome and Gene Expression Profiling of SiHD-ZIP Class I Entailing Drought Stress Responses at Early Seedling Stage
}

\section{Maryam Mehmood}

COMSATS University Islamabad

Muhammad Jadoon Khan

COMSATS University Islamabad

Muhammad Jawad Khan

COMSATS University Islamabad

Nadeem Akhtar

COMSATS University Islamabad

Fizza Mughal

University of Illinois at Urbana-Champaign

Syed Tahir Abbas Shah

COMSATS University Islamabad

Muhammad Zeeshan Hyder

COMSATS University Islamabad

Sumaira Farrakh ( $\nabla$ sumaira.farrakh@comsats.edu.pk)

COMSATS University Islamabad

Irfan Sadiq

COMSATS University Islamabad

\section{Research Article}

Keywords: HD-ZIP, Transcription factors, Sesamum indicum, Queen of seeds, Drought tolerance

Posted Date: June 15th, 2021

DOl: https://doi.org/10.21203/rs.3.rs-592783/v1

License: (1) This work is licensed under a Creative Commons Attribution 4.0 International License.

Read Full License 
Version of Record: A version of this preprint was published at Molecular Biology Reports on January 7th, 2022. See the published version at https://doi.org/10.1007/s11033-021-07024-2. 


\title{
Systematic analysis of HD-ZIP transcription factors in sesame genome and gene expression profiling of SiHD- ZIP Class I entailing drought stress responses at early seedling stage
}

\author{
Maryam Mehmood ${ }^{1}$. Muhammad Jadoon Khan ${ }^{1}$. Muhammad Jawad Khan ${ }^{1}$. Nadeem Akhtar ${ }^{1}$. Fizza Mughal ${ }^{2}$. Syed \\ Tahir Abbas Shah ${ }^{1}$. Muhammad Zeeshan Haider ${ }^{1}$. Sumaira Farrakh ${ }^{1 *}$. Irfan Sadiq ${ }^{*}$ \\ 1. Department of Biosciences, COMSATS University Islamabad, Park road, Islamabad, Pakistan \\ 2. Illinois Informatics Institute, University of Illinois, Urbana-Champaign \\ *Correspondence: \\ Dr. Irfan Sadiq \\ Department of Biosciences, COMSATS University Islamabad, Islamabad, Pakistan \\ Email: irfan.sadiq@fulbrightmail.org \\ Dr. Sumaira Farrakh \\ Department of Biosciences, COMSATS University Islamabad, Islamabad, Pakistan \\ Email: sumaira.farrakh@comsats.edu.pk
}

Key message

- Drought is a key abiotic stress affecting sesame growth and productivity

- HD-ZIP transcription factors, one of the most widely studied proteins in plants drought responses, particularly HD-ZIP Class I genes

- The differential gene expression of SiHD-ZIP Class I genes in drought leads to various changes at early seedling stage in sesame

- The SiHD-ZIP Class I genes can be used to elucidate drought tolerance mechanisms in sesame

\begin{abstract}
Sesame is a very ancient oilseed crop. Sesame sensitivity to drought stress at early seedling stage is one of the limiting factors affecting its growth and yield in the world. HD-ZIP transcription factors family is one of the most important families involved in drought stress responses in plants. In this study, total sixty one sesame HD-ZIP (SiHZ) proteins were identified in sesame, based on protein sequence homology with Arabidopsis and protein domain(s) architectures were predicted by Hidden Markov model (HMM). HD-ZIP proteins were then classified into four classes (HD-ZIP Class I-IV) according to the phylogenetic, conserved domain(s) motifs and gene structure analyses in sesame. Based
\end{abstract}


on comparative phylogenetic analysis of sesame with Arabidopsis and maize HD-ZIP protein sequences, HD-ZIP Class I was subdivided into four subgroups $\alpha$ (SiHZ25, SiHZ43, SiHZ9 and SiHZ16), $\beta 1$ (SiHZ10, SiHZ30, SiHZ32 and SiHZ26), $\beta 2$ (SiHZ42 and SiHZ45) and $\square \square(\mathrm{SiHZ17,SiHZ7} \mathrm{and} \mathrm{SiHZ35).} \mathrm{Twenty-one} \mathrm{days} \mathrm{old} \mathrm{Sesame} \mathrm{seedling}$ were exposed to severe drought stress by withholding water for 7 days. Gene expression of 13 members of HD-ZIP Class I was performed in well- watered (control) and water stressed (treatment) seedlings. The results of gene expression analysis showed that, SiHZ7 (6.8 fold) and SiHZ35 (2.6 fold) from $\square \square$ subgroup showed significantly high gene expression levels under drought stress in sesame seedlings. Thus, this study provides useful molecular information pinpointing the role SiHD-ZIP Class I in drought stress responses at early seedling stage and to develop sesame novel varieties with improved drought tolerance in sesame.

Key words: HD-ZIP. Transcription factors. Sesamum indicum. Queen of seeds. Drought tolerance.

\section{Funding}

The authors did not receive support from any organization for the submitted work

\section{Conflicts of interest/Competing interests}

The authors declare no conflicts of interest.

\section{Acknowledgements}

We extend our gratitude to the Translational Genomics lab at the Department of Biosciences, CUI, especially Dr. Azeem Butt, for providing us a platform to perform our gene expression analysis on this work.

\section{Availability of data and transparency}

NA

\section{Code availability}

Not applicable

\section{Authors' contributions}

Maryam Mehmood. Performed all the experiments and wrote the manuscript, Muhammad Jadoon Khan: Helped in gene expression analysis. Muhammad Jawad Khan: Helped in Real time related work. Nadeem Akhtar: 
Helped in Bioinformatics analysis. Fizza Mughal: Computational assistance in HD-ZIP transcription factors identification. Syed Tahir Abbas Shah: Helped in statistical analysis. Zeeshan Haider: Technical help and provided lab facility. Sumaira Farrakh: supervise research, helped in finalizing the manuscript. Irfan Sadiq: conceived the idea, plan the research and finalize the manuscript.

\section{Ethics approval}

Not applicable

\section{Consent to participate}

Not applicable

\section{Consent for publication}

Not applicable

\section{Introduction}

Sesame (Sesamum indicum L., 2n= 26) is a very ancient oil seed crop [1]. It is lauded as the "Queen of Seeds" as its seeds are a rich source of high quality and highly stable edible oil (50-60\%) [2]. It is essential to human life in several ways serving as a good source of proteins (25\%), carbohydrates (13.5\%), polyunsaturated fatty acids, lignans (sesaminol sesamin, sesamol and sesamolinol), vitamins (B \& E) and several mineral ions (phosphorous, iron, magnesium calcium, manganese, copper, and zinc) [3, 4]. Due to the pleasing aroma and taste of its seeds and oil, it is widely used for multiple purposes like cooking, confectionary, baking, livestock, medicines, and cosmetics [5]. Sesame is cultivated in arid and semi-arid lands, but its growth and yield are substantially reduced as compared to other economically important oil crops (e.g. rapeseed, peanut and soya bean) due to high temperatures and episodic/terminal drought conditions [6,7]. Although, sesame is a well-known "survivor" or "drought tolerant" oil crop yet it is highly sensitive to drought, particularly at germination and flowering (anthesis) stage, which ultimately affect its growth and yield [3].

The identification and development of drought tolerant varieties is a crucial to sustain production of sesame crop. However, for the development of drought tolerant varieties, it is very important to understand drought tolerance mechanisms [7]. So far, only a handful molecular studies can be found on sesame drought responses, particularly at 
early growth stage. Transcription factors (TFs) are one of the most important gene families participating in transcriptional regulation of drought stress responses in sesame [1].

Homeodomain-leucine zipper (HD-ZIP) is a well-characterized TF family specific to plant species. It is related to growth, development, and stress responses in plants [8]. It belongs to a very large gene family, well-known as homeobox (HB) [9]. It is characterized by a highly conserved region ( 60 amino acid sequence) known as homeodomain (HD) for specific DNA binding and less conserved motif, called leucine zipper (LZ) for protein-protein interactions through homo- and/or hetro-dimerization [10]. HD-ZIP TF family is mainly divided into four classes (HD-ZIP Class I-IV) in plants [11-13].

HD-ZIP Class I which is characterized by highly conserved HD-domain and less conserved LZ motif, serves as a binding-site for dyad-symmetric sequence CAAT(A/T)ATTG $[14,15]$. This class has crucial roles in plant growth, development and in abiotic stress responses. Moreover, it is also reported to be linked with cell membrane stability and abscisic acid (ABA) signaling leading to reduced plant growth specifically under drought stress [10, 13]. HD-ZIP Class II possesses CPSCE (Cys, Pro, Ser, Cys, Glu amino acids) motif and N-terminal consensus sequence, in addition to well-conserved HD-domain and LZ motif [16, 17]. Like Class I, Class II members also bind to CAAT-N-ATTG sequence but differ in the recognition of nucleotide located in the center of the target site [16]. This class is particularly involved in plant growth and development, shade avoidance, auxin response and drought stress [11]. In contrast, HDZIP Class III is distinguished from other HD-ZIP classes by having START domain (steroidogenic acute regulatory protein-related lipid transfer), an adjacent conserved region SAD (START-adjacent domain) and MEKHLA at Cterminal along with HD-domain and LZ motif $[18,19]$. Based on in-vitro studies, HD-ZIP Class III recognizes GTAAT(G/C)ATTAC sequence as binding-site [14]. It is mainly involved in plant growth/development and oxygen redox/light signaling processes [18]. In contrast to Class I and II, role of Class III is least understood in abiotic stress like drought, salt stress and ABA treatment [11]. In HD-ZIP Class IV, HD-domain and LZ motif (loop in the middle) are also present as in Class I-III [10]. HD-ZIP Class IV also possesses START domain (lipid binding domain) like Class III which binds to AAATG(C/T)A but lacks MEKHLA domain [14]. Like Class III, Class IV has no role in abiotic stresses rather it bears functional importance in cell differentiation, flowering and cuticle development [11].

In plants, HD-ZIP Class I of HD-ZIP TF family involved in abiotic stresses, including drought. HD-ZIP Class I is further categorized into six monophyletic subgroups or clades $(\alpha, \beta, \gamma, \delta, \varepsilon$, and $\varphi)$ in Arabidopsis [14]. HD-ZIP Class 
I TFs (like Zmhdz10 in maize) were induced by ABA treatment in drought stress and regarded as the positive transcriptional regulators to confer drought tolerance in plants [20-22]. As evident from previous studies, HD-ZIP Class I genes from $\gamma, \beta$ and $\alpha$ clades showed notable response to drought stress in different plant species such as Arabidopsis [23] and maize [24]. In fact, depending upon plant developmental stage and environmental conditions, different gene members of this class exhibit different gene expression levels during drought stress [10]. The expression of genes from $\square$-clade - AtHB7, and AtHB12 (Arabidopsis), OsHOX6, OsHOX22, and OsHOX24 (rice), HaHB4 (sunflower), MtHB1 (Medicago) and NaHD2O (tobacco) - is typically high in drought stress demonstrating reduction in plant growth [25]. Similarly, upregulation of ATHB5, -6, -7 and -12 ( $\beta$-clade) in Arabidopsis [14] and Zmhdz7, Zmhdz1, Zmhdz3 and Zmhdz2 ( $\beta$-clade) in maize is also evident from previous studies under drought stress and ABA treatment [26]. In contrast to these, downregulation of Zmhdz15 ( $\beta$-clade) under drought stress and in ABA treatment was reported in maize [26]. Like HD-ZIP I $\beta$-clade, gene expression of HD-ZIP $\alpha$-clade can also be increased (AtHB13 and $H a H B 1)$ or decreased (Zmhdz11, Zmhdz5 and Zmhdz13) upon drought stress [10].

The genes of HD-ZIP Class I (wheat TaHDZipI-3, -4 and -5) were characterized to specifically bind to stress-related cis-elements in the promoter sequences of drought-responsive genes to regulate drought responses [22]. In addition to this, these genes might act as upstream regulator of other TFs conferring drought tolerance in plants [10]. This type of association was previously reported between AtHB13 (HD-ZIP Class I) and JUB1 (a member of NAC TF family), establishing a joint drought stress control module as an upstream regulator of $J U B 1$ and later as a positive regulator of drought tolerance in Arabidopsis [10]. Moreover, altered gene expression of stress/ABA-responsive genes such as D1-Pyrroline-5-carboxylate synthetase 1 (P5CS1), Responsive to dehydration 22 (RD22), Responsive to dehydration $29 B(R D 29 B)$ and $A B A$ insensitivel $(A B I 1)$ is another attribute of HD-ZIP Class I mediated drought stress tolerance, as reported in Zmhdz10 over-expressors transgenic rice plants [21].

So far, genome-wide analyses have been performed on a number of stress related TF families such as ERF, WRKY, MYB, NAC and bZIP in sesame [27]. Moreover, genome-wide classification of HD-ZIP gene family and their expression analysis under drought and salinity stress in different tissues (root, stem, flower, leaf, capsule and seed) of sesame has already been reported [27]. In the current study, the classification of HD-ZIP proteins in sesame has not only extended from its four well-conserved classes to further subgrouping of Class I into and $\square \square \square$ but also possible role of $\square \square \square \square \square \square \square \square \square \square$ and $\square \square \square$ was explored in severe drought 
stress response of sesame at early stage. To our knowledge, the role of HD-ZIP Class I in drought stress responses entailing early growth stages in sesame has not been studied so far.

\section{Materials and Methods}

\section{In silico identification of SiHD-ZIP proteins}

HD-ZIP protein sequences were identified from sesame whole proteome ( $S_{-}$indicum_v1.0), downloaded from National Center for Biotechnology Information (NCBI: https://www.ncbi.nlm.nih.gov/genome/). This identification was based on typical homology search and prediction of protein domain architecture using The HMMER approach. First of all, homology search was performed between sesame whole proteome sequences with that of protein sequences of A. thaliana transcription factors families, retrieved from The Arabidopsis Information Resource (TAIR) (https://www.arabidopsis.org/) to create a database for homology search on BLAST standalone version (2.6.0+). The candidate TFs orthologs in sesame were predicted based on reciprocal BLASTP hits defined as: $\geq 80 \%$ query coverage, e-value $<10^{-6}$ and $\geq 50 \%$ sequence identity. From the set of identified TFs orthologs in sesame, redundant protein data (duplicate hits) was eliminated using python scripting. Thus, obtained non-redundant protein sequences with query coverage $\geq 80 \%$ were selected as candidate transcription-associated proteins (TAPs) in sesame. Candidate TAPs were verified based on their domain(s) architecture using Superfamily (1.75) based on HMM library comprising Hidden Markov's Models [12] and python scripting. After domain(s) verification, the proteins were classified into plantspecific TF and TR families in sesame, according to a consensus rule based on previously established methods for domain(s) assignment in protein sequences, as reported by [28]. Total number of well-characterized HD-ZIP proteins as well as their respective gene loci, linkage groups (LG1-LG16), chromosomal locations and exon counts from NCBI were determined in sesame. Subsequently, based on this information, a numerical name was designated to each of these predicted SiHD-ZIP protein sequences depending upon their distribution order (LG1-LG16) in sesame.

\section{Phylogenetic analysis and classification of SiHD-ZIP proteins}

After removing unassigned and outlier protein sequences from the analysis, the predicted SiHD-ZIP proteins were selected for their classification into four well-conserved classes of HD-ZIP TF family known in plants. Multiple sequence alignments were built by ClustalW (http://www.clustal.org/). Then, a phylogenetic tree was constructed by choosing maximum likelihood (ML) method and 1000 bootstrap replications in order to divide them into four classes (SiHZ I-IV) using MEGA (version X) [29]. For the sub-classification of SiHD-ZIP Class I into subgroups and clades, second ML tree was built from the multiple sequence alignment of protein sequences of SiHD-ZIP Class I and already- 
reported members of SiHD-ZIP Class I in maize and Arabidopsis [26]. After construction, the phylogenetic trees were analyzed using Dendroscope version 3.7.2 [30].

Identification of conserved domain(s), isoelectric point (pI)/molecular weight (kDa) and isoelectric point (pI) in SiHD-ZIP Class I-IV

To validate the classification of SiHD-ZIP Class I-IV protein sequences, the conserved motifs of these proteins were determined by subjecting protein sequences to online MEME (Multiple Expectation Maximization for Motif Elicitation) version 5.1.1 with parameters set as: optimum motif width $(\geq 6$ and $\leq 200)$; maximum number of motifs (20) [31]. The conserved motifs were then further verified by two conserved motifs prediction tools, Expasy PROSITE [32, 33] and Interpro 82.0 [34]. Moreover, domain architectures of HD-ZIP Class I-IV proteins were also predicted by SMART (Simple Modular Architectural Research Tool), to confirm the presence of class-specific domains in SiHD-ZIP protein sequences [35]. The isoelectric point $(\mathrm{pI}) /$ molecular weight $(\mathrm{kDa})$ of each of these proteins were calculated by Expasy online tool https://web.expasy.org/compute_pi/.

\section{Gene structure analysis of SiHD-ZIP proteins}

The gene structure analysis of SiHD-ZIP Class I-IV was performed by Gene Structure Display Server (GSDS) version 2.0 [36]. To determine their gene structures, the coding and genomic sequences of these proteins were obtained from NCBI database.

\section{Plant material and drought stress treatment}

Sesame seeds of TS-5 variety were collected from National Agricultural Research Centre (NARC), Islamabad, Pakistan. After overnight soaking ( 24 hours) in fresh water, seeds were dried and sown in plastic pots filled with a mixture of soil, sand and peat in 2:1:1 ratio and kept at $25 \pm 3^{\circ} \mathrm{C}$ with 16 -h day, 8 -h night photoperiod. After 20 days of seedling growth, drought stress was imposed by complete water withholding in drought treated seedlings. The control plants were watered regularly throughout the experiment. The leaf samples were subsequently collected from both control and drought stressed seedlings when the soil moisture reached $16 \%$. After collection, each leaf sample was immediately treated with liquid nitrogen for subsequent RNA extraction and qPCR. The experiment was performed with three independent biological replicates.

RNA extraction and quantitative polymerase chain reaction (qPCR) analysis 
For the gene expression analysis of SiHD-ZIP Class I genes under drought stress, total RNA was extracted from the leaf samples of control and drought stressed seedlings using Trizole method [37]. The integrity of the isolated RNA was assessed by $1 \%$ denaturing agarose gel. The concentration of isolated RNA was determined by a spectrophotometer (Analytik Jena SPECORD 200 plus). After RNA quantification, each sample was further processed with DNase 1 (Thermo Fisher Scientific) to remove DNA contamination. The DNA-free RNA sample was converted into cDNA by using a cDNA synthesis kit (Thermo Fisher Scientific). Gene-specific primers for qPCR were designed for the identified genes of HD-ZIP Class I by using the NCBI primer designing tool (https://www.ncbi.nlm.nih.gov/tools/primer) and are listed in Supplementary Table S3. The qPCR was carried out using Maxima SYBR Green/ROX qPCR Master Mix (Thermo Fisher Scientific) at $54{ }^{\circ} \mathrm{C}$. The actin gene was used as reference gene and three independent biological replicates for control and drought stressed seedlings were used to carry out the gene expression analysis by qPCR. Relative fold-change of target genes was determined by $2^{-\square \square C t}$ method as described by [38].

\section{Statistical analysis}

For statistical analysis one-way ANOVA was performed followed by Tukey's test at significance level $\mathrm{P}=0.05$ using Origin 2019b (https://www.originlab.com/index.aspx?go=Products/Origin/2019b\&pid=3322). The results were expressed as mean \pm standard error (SE) of three replicates.

\section{Results}

\section{In-silico identification of SiHZ proteins}

In the given study, total 61 well-annotated HD-ZIP proteins were predicted in sesame genome. After prediction, names were assigned to these protein sequences ( $\mathrm{SiHZ} \mathrm{1-45)} \mathrm{according} \mathrm{to} \mathrm{their} \mathrm{chromosomal} \mathrm{locations} \mathrm{on} \mathrm{sesame} \mathrm{linkage}$ groups (LGs) (Supplementary Table S1).

\section{Phylogenetic analysis of HD-ZIP proteins and their classification in sesame}

Based on the phylogenetic analysis, the identified HD-ZIP protein sequences were classified into four classes (Figure 1). The resulting phylogenetic tree (Maximum likelihood (ML) tree) reveals the distribution of selected SiHZ proteins into four classes: Class I (13), Class II (12), Class III (21) and Class IV (14) (Fig. 1). 
Subsequently, an ML tree was also constructed for further division of HD-ZIP Class I into its clades or sub-groups $(\alpha$, $\beta 2, \beta 1, \zeta, \delta, \varepsilon, \gamma$ and $\varphi)$ as shown in Fig. 2. In Class I, the highest number of protein members fall into $\square \square$ subgroup (6). Out of these six members, SiHZ10, SiHZ30, SiHZ32 and SiHZ26 were clustered in $\square \square 1$ while SiHZ42 and SiHZ45 in $\square 2$ (Fig. 2). Besides these, four members (SiHZ25, SiHZ43, SiHZ9 and SiHZ16) were included in sub-group followed by three members (SiHZ17, SiHZ7 and SiHZ35) in $\square$ sub-group (Fig. 2). None of the members of Class I were found in subgroups $\zeta, \delta, \varepsilon$ and $\varphi$ in sesame (Fig. 2).

\section{Sesame HD-ZIPs conserved domains, their isoelectric points (pI/molecular weight, MW (kDa) in Class I-IV}

In sesame, for validation of HD-ZIP proteins (Class I-IV), their conserved domains were identified by MEME tool (Fig. 3). Total 20 conserved domains were identified in $\mathrm{SiHZ}$ proteins, ranging from 20 to 98 amino acids in length (T). Then, conserved amino acid sequences of these domains were used to confirm domain's presence and its molecular function in all four classes with the help of Expasy Prosite and InterPro (Supplementary Table S2). From Expasy Prosite, only Homeobox domain was predicted in Motif 1 (common feature of all four classes of HD-ZIP proteins) and START domain in Motif 4, which is a characteristic feature of both, Class III and IV (Supplementary Table S2). The selected protein sequences were analyzed with InterPro. Analysis by InterPro showed greater number of predicted conserved domains and their molecular function to validate HD-ZIP proteins in all four classes in sesame i.e. Motif 1, 3, 4, 5, 8, 9 and 11 (Supplementary Table S2). The presence of specific domains belonging to four classes was confirmed by SMART (Fig. 4). According to results, HOX domain was found in all Classes (I-IV), HALZ domain was exclusively identified in Class II, START domain was found both in Class III and IV but MEKLA domain was only present in Class III (Fig. 4).

Furthermore, number of amino acids (a.a), isoelectric point (pI)/molecular weight (MW) of these selected proteins were also analyzed. The result showed great variation among member proteins belonging to each class in their amino acids sequence length, molecular weight and isoelectric point (Table 1). In sesame, HD-ZIP proteins ranged from a minimum of 201 ( $\mathrm{SiHZ7}$ from Class I) to a maximum of 853 (SiHZ41b from Class III) amino acids in length. Among SiHZ proteins, the largest amino acids sequence length was obtained in Class III ranging from 803 a.a in SiHZ22c to 853 a.a in SiHZ41b while the shortest range was found in the members of Class I from 201 a.a (SiHZ7) to 340 a.a (SiHZ30). Likewise, the pI values were high in Class III and ranged from 5.81 (SiHZ34) to 6.28 ( $\mathrm{SiHZ13}$ ) as compared rest of the classes. In Class IV, it ranged from 5.40 (SiHZ6a, b) to 8.50 (SiHZ12b) while in Class II ranging from 4.99 
(SiHZ3a, b,) to 8.74)] and 4.73 (SiHZ10) to 7.71 (SiHZ7) in Class I (Table 1). Similarly, the range of molecular weights (kDa) was determined highest in Class III members followed by Class IV, II and I (Table 1).

\section{Sesame HD-ZIPs chromosomal locations and their gene structure analysis in Class I-IV}

In sesame, an uneven distribution of SiHD-ZIP genes was found, located over 1-12 LGs (Table 1). Among these genes, total 13 genes of Class I were unevenly distributed on six different LGs (2, 4, 8, 11, 13 and 15) while 10 genes of Class II were located on LGs 1-4, 6, 11 and 15 (Table 1). Similarly, genes in Class III and IV, each comprising 8 and 9 genes, respectively, were located on LGs (1,3,4,6-9 and 12) and (1-4 and 8), respectively (Table 1). The greatest number of genes (9) in Class IV were found distributed over lesser number of LGs (1-4 and 8) as compared to Class I-III (Table 1). Moreover, LG 4 was found common to all classes having two genes from Class I and one of the gene members of Class II-IV (Table 1). Besides this, LG 13 was only common to Class I and LGs (7, 9 and 12) were only identified in Class III (Table 1).

Likewise, the gene structure analysis of SiHD-ZIP genes showed great variability in the number of introns ranging from 1-17 (Fig. 5). The results showed genes of each class were quite similar in exon-intron structure and clustered together. The number of introns in Class I and II were comparatively lesser than Class III and IV. In Class I, majority of the genes had two introns except for SiHZ 7, SiHZ17 and SiHZ35 which had only one intron in their genes and SiHZ26 had three introns. Likewise, most of the genes had three introns in Class II but SiHZ14 and SiHZ44 were the genes with two introns. Out of these genes, the highest number of introns (17) was found in genes of the Class III while the number of introns in Class IV genes was comparatively greater than Class I \& II but lower than Class III, ranging from 8-10 introns (Fig. 5).

\section{Effect of drought stress on sesame seedlings}

Under severe drought stress condition, sesame seedlings showed reduced or slow growth as compared to control (wellwatered) seedlings in sesame (Fig. 6). In addition to slow plant growth, curling of leaves with mild yellowing were also observed in drought treated seedlings (Fig. 6).

\section{Expression analysis of HD-ZIP Class I genes under drought stress at early seedling stage in sesame}

In this study, gene expression analysis of 13 genes of SiHD-ZIP Class I was performed in seedling under severe drought stress along with well-watered control. Out of these 13 genes, 8 genes showed differential expression under 
drought stress. The results demonstrate significantly high upregulation of two genes $\mathrm{SiHZ7}$ (6.8 fold) and $\mathrm{SiHZ35}$ (2.6 fold) from $\square \square$ subgroup while downregulation of two other genes (SiHZ25 with fold change $\sim 0.76$ and SiHZ43 having fold change $\sim 0.59$ ) of $\square \square$ subgroup (at $\mathrm{P}<0.05$ ) in severe drought stressed as compared to control seedlings (Fig. 7). In addition to this, the expression of rest of the selected genes i.e. SiHZ9 and SiHZ16 ( $\square$ ); SiHZ10, SiHZ26, SiHZ30 and SiHZ32 ( $\square$ 1); SiHZ42 and SiHZ45 ( $\square 2)$ and $\operatorname{SiHZ17}(\square)$ showed no significant difference in the gene expression levels between drought stressed and control seedlings (Fig. 7).

\section{Discussion}

\section{In-silico identification of SiHD-ZIP proteins}

HD-ZIP is a plant-specific TF family known for its roles in plant growth, development and responses to light and stress [39]. So far, HD-ZIP is a widely characterized TF family throughout plant kingdom such as Arabidopsis (48) [14], rice (48) [40], maize (55) [26], wheat (46) [22], potato (43) [39], tomato (51) [41], medicago (52) [42], foxtail millet (47) [20], tea plant (33) [8], grape (31) [43], cassava (57) [11], soybean (88) [44], Chinese cabbage (113) [45], poplar (63) [46], pear (52) [47], peach (33) [48], plum blossom (32) [49] and eucalyptus (40) [50]. In the given study, total 61 sesame HD-ZIP proteins, encoded by comparatively smaller number of genes (44) have been identified, based on sequence homology search (A. thaliana) and domains assignment rules by HMM (Supplementary Table 1). In the light of previous studies, results showed that species bias does exist in the distribution of HD-ZIP genes and it varies from one plant species to another [46]. The HD-ZIP genes are most likely to evolve and expand in numbers due to segmental gene duplication events in different plant species [27]. Thus, the number of HD-ZIP genes in sesame (44) found in this study was almost similar to that of potato (43) [39]. In contrast to this, the highest number of HD-ZIP genes has been reported in Chinese cabbage (113) [45] and soybean (88) [44, 51]. Thus, these findings shed light on the evolutionary history of sesame as well. From evolutionary point of view, it is distantly related to other oil crops such as soybean, castor and rape but closely related to potato (Solanaceae family) and Phrymaceae families in the core lineage of eudicots-angiosperms [52]. Thus, the number of HD-ZIP genes in sesame sharing similarity with potato but greatly differing from soybean is providing an interesting clue about the evolutionary relationship of sesame with other oilseed crops.

\section{Phylogenetic analysis of SiHD-ZIP proteins and their classification}


In this study, a phylogenetic analysis was performed for the classification of sesame HD-ZIP proteins into four wellconserved classes known in plants (Fig. 1). The clustering of similar HD-ZIP members within the same class in sesame is clear from this phylogenetic analysis (Fig. 1). These results clearly reflect high conservation of HD-ZIP TF family in plants and agree with previously classified HD-ZIP TFs in other plant species like Arabidopsis [14], maize [26], potato [39] and eucalyptus [50].

In addition to this, sesame HD-ZIP Class I was further divided into subgroups in this study. The subgroups assignment of member proteins in Class I was done based on their clustering within already known subgroups $(\alpha, \beta 2, \beta 1, \zeta, \delta, \varepsilon$, $\gamma$ and $\varphi$ ) in Arabidopsis and maize (Fig. 2). According to results, clades $\alpha, \beta 2, \beta 1$ and $\gamma$ were common to all three plant species selected for the phylogenetic analysis. In previous studies, some of these subgroups were shown to be lineagespecific such as $\square \square$ clade (subgroup) is exclusively shared among eudicots (Arabidopsis, Medicago, grape, and poplar) while clade $\square \square$ is common to monocots (rice, millet, and stiff brome) and $\square$ clade $\square \square$ to moss [46]. It is clear from these results that these subgroups were neither found in sesame nor Arabidopsis and maize. In addition to these, sesame also lacks clade $\delta$ which was common to both Arabidopsis and maize as well as clades $\varepsilon$, and $\varphi$ which were only common to Arabidopsis [24]. So, the subgrouping within the Class I found to be consistent with previously reported studies. In Fig. 2, the presence of $\square 1$ clade and absence of clade $\square \square \square$ in sesame and Arabidopsis as compared to maize (monocot) ensures their eudicot lineage. It is most likely that sesame might share more functional similarity with Arabidopsis HD-ZIP I class in transcriptional regulation of genes during growth, development and/or abiotic stress responses, including drought.

\section{SiHD-ZIP Class I-IV conserved domains and their isoelectric points (pI)/ molecular weight (Da)}

In the given study, high consensus was found in the conserved domain(s) architectures of member proteins within each class, represented by their respective member as shown in Fig. 6. According to results, the domain(s) architectures were common to all members within each class of SiHD-ZIPs and were supported by previous findings [53, 54]. Likewise, all SiHD-ZIP members within each class shared great similarity in the values of their amino acid number, pI/MW (Table 1). Overall, members of Class I and II have shorter amino acids sequence length as compared to Class III and IV (Table 1). Similarly, there was more difference in pI values within members of Class I and II as compared to Class III and IV in sesame. Thus, these results clearly reflect same motif organization within the same class but different motif organization among all four classes in SiHD-ZIPs. Hence, these results clearly demonstrate classspecific diversity in the protein sequence, structure and function of HD-ZIP TF family in sesame. 


\section{SiHD-ZIP Class I-IV chromosomal locations and their gene structure analysis}

According to results, there was an uneven distribution of SiHD-ZIP genes located on 1-12 LGs in sesame (Table 1). These results represented the highest number of gene distribution on LG 8 [10 genes: Class I (3), Class III (1) and Class IV (5)] in sesame (Table 1). In contrary, the lowest gene distribution of $H D$-ZIP genes was found on LGs 7, 12 and 13 i.e. only one gene per LG (Table 1). Moreover, the gene structure analysis of SiHD-ZIP genes also revealed similar gene structure organization (gene size, number of introns and exons as well as their lengths) shared among members of each class (Fig. 5). Thus, these findings clearly suggest that SiHD-ZIP genes located on the same LG (chromosome) are most likely to be linked with each other. Thus, it is inferred that these linked genes might function together in transcriptional regulatory gene networks involving plant growth, development, metabolic pathways and/or or stress responses in sesame.

This is another inevitable finding of this study that the number of SiHD-ZIP genes (44) identified in this study was quite close to the previously reported number of SiHD-ZIP genes (45) in sesame (45) [27]. According to results, the number of identified SiHD-ZIP proteins (61) was much greater than the number of total genes (44) in sesame (Table 1). In this respect, HDZIP Class III is the largest class having the greatest number of SiHD-ZIP proteins (21) encoded by only 9 genes in sesame (Table 1). Similarly, Class IV has total 14 protein members, encoded by only 7 genes and Class II comprises of 12 member proteins encoded by 10 genes (Table 1). In contrast to them, the 13 member proteins are encoded by 13 different genes in Class I (Table 1). Such comparative analysis between SiHD-ZIP proteins (splice variants or protein isoforms) and their respective genes provides useful information to elaborate the functional diversity of HD-ZIP TF family and organism's complexity in sesame [55, 56]. So, in the given study, some of the HDZIP proteins in sesame are possible variants (protein isoforms) of the same $H D$-ZIP gene which can be formed at a time depending upon the tissue type or stage of development [57]. These results are consistent with previously reported studies e.g., the number of $H D$-ZIP genes found in potato were 15, 10, 7 and 9 in Class I-IV, respectively [39], which is quite similar to the results reported in this study. In contrary, Arabidopsis and maize, both of them have the least number of genes (5) in HD-ZIP Class III while more genes members belong to HD-ZIP Class II (18) and HD-ZIP Class I (17) followed by HD-ZIP Class IV (15) in Arabidopsis and 17, 16 and 10 in Class I, IV and II, respectively, in maize [26]. Moreover, a good comparison can be made between SiHD-ZIP Class I-IV proteins and soybean in which the largest number of HD-ZIP proteins falls in Class I (30) and II (27) while least in the Class III (12) and 19 
in class IV soybean [51]. Thus, if the comparison of these classes is made based on number of genes, more structural and functional diversity can be linked in the genes of Class I. Thus, the functional diversity in sesame's HDZIP TF family can be implicated by two means, either through the expansion of the Class I by segmental gene duplication events [27] or alternative splicing of genes to produce multiple splice variants (protein isoforms) from a single or few genes of Class III, IV and II respectively [56].

\section{Effect of drought stress on sesame seedling growth}

It is evident from results that sesame seedlings did not show wilting rather resumed slow plant growth with slight yellowing and curling of leaves (Fig. 6). As evident from previous findings, this reduction in growth can be linked to some molecular alterations (e.g. over-expression of member gene(s) from HD-ZIP Class I) to confer drought tolerance in sesame at seedling stage [53].

\section{Expression analysis of HD-ZIP Class I genes under drought stress at early seedling stage in sesame}

The functional relevance of SiHD-ZIP Class I in drought and other abiotic stress responses is widely reported in several plant species like Arabidopsis [53], sunflower [58] and tobacco [59]. Recently, significantly high expression of HD-ZIP I genes (MtHDZ17, MtHDZ34, MtHDZ35, MtHDZ39, MtHDZ47, MtHDZ48, and MtHDZ50) along with several members of HD-ZIP Class II has been reported in response to drought and salinity stress treatments in medicago [42]. However, changes in HD-ZIP Class I gene expression are inevitable, induced by water-limiting conditions or ABA treatment. In cassava, a dramatic change in the expression of Class I genes in leaves under drought stress suggests longer periods of drought could cause dramatic effects on cassava [11]. Similarly, both, upregulation or downregulation of Class I genes at different time points (6hr, $12 \mathrm{hr} \& 24 \mathrm{hr})$ have also been reported in soybean [44]. According to the results of this study, members of Class I showed either upregulation, downregulation or no change in their gene expression in the drought treated seedlings compared to the control (well-watered) seedlings (Fig. 6). Similar pattern of gene expression was observed in previous studies in other plants such as tobacco, where HD-Zip class 1 genes showed similar trends in response to drought stress [59].

In the given study, SiHZ7 and SiHZ35, two members of subgroup $\square \square$ subgroup exhibited significant upregulation under drought stress. Similarly, as previously reported in maize, the gene expression levels of subgroup $\gamma$ (Zmhdz12, $-9,-6$ and -4 ) were strongly induced by more than 2-fold compared to the control [26]. Such high gene expression of members from $\square \square$ clade is typically induced by ABA treatment and water deficit conditions and have previously reported in Arabidopsis (AtHB7, AtHB12), sunflower (HaHB4), medicago (MtHB1), tobacco (NaHD20), maize 
(ZmHDZ4) and rice (OsHOX6, OsHOX22, and OsHOX24) enhancing drought and salinity tolerance via ABA signaling in transgenic plants [27]. Albeit, SiHZ7 and SiHZ35, are the two members of the same subgroup $\square$, showed high gene expression levels under drought stress in this study. They share great similarity with each other as two paralogs yet their divergent evolution and function can be speculated in order to mediate growth responses under water deficit conditions as previously reported in Arabidopsis (AtHB7 and AtHB12) [60,61]. In the light of previous findings, reduction in cell elongation and plant growth can be inferred with the upregulation of $\mathrm{SiHZ7}$ and $\mathrm{SiHZ5}$ in sesame under drought stress [53]. Most probably, this reduction in cell elongation and subsequent drought tolerance in sesame can be either due to inhibition of GA biosynthesis such as AtHB12 in Arabidopsis or without the inhibition of GA biosynthesis like $A t H B 7$ in Arabidopsis) [53]. In addition to this, like AtHB13 (Arabidopsis), either both or any of these two TFs (SiHZ7 or SiHZ35) may confer drought tolerance at early seedling stage by acting as upstream positive regulator(s) of other TFs such as NAC (JUB1 or its homolog) in sesame [53]. Thus, it can be speculated that $\mathrm{SiHZ7}$ and SiHZ35 may work in a coordinated fashion by changing their gene expression levels to fine tune processes associated with growth (early seedling stage) and water stress in sesame [62]. However, functional analysis of SiHZ7 and $\mathrm{SiHZ35}$ is required to draw a better picture of gene or metabolic network(s) involved in sesame drought stress responses/tolerance at early seedling stage.

On the other hand, two members of subgroup $\square$ (SiHZ25 and SiHZ43) showed their downregulation in drought seedlings as compared to control. These results seem quite similar with the expression of HDZ-I genes from-clade in maize [24]. According to [24], drought stress resulted in the downregulation of Zmhdz11, -5 and -13 of subgroup $\square \square \square$ while the expression levels of the subgroup $\gamma$ genes (Zmhdz12, -9, -6 and -4 ) were strongly induced more than 2-fold as compared to the control plant [24]. In contrast to this, AtHB13 (Arabidopsis) and HaHBI (sunflower) were found to constitutive overexpression of $\square \square \square$ clade members with no significant effect on the growth and yield of transgenic Arabidopsis under drought stress [63]. However, their high expression levels may induce plant stress tolerance to cold, drought and/or salinity by enduring cell membrane integrity and stability in plant cells [63]. In the given study, no significant gene expression changes were found in case of subgroup $\square$ under drought stress, particularly, SiHZ26 and SiHZ32 ( $\square$ 1) and SiHZ42 ( $\square 2)$. In contrast to these results, either up- or downregulation of subgroup $\square$ gene members is evident in different plant species from previous studies. In Arabidopsis, elevated levels of $A T H B 5, A T H B 6, A T H B 7$, or $A T H B 12$ were induced in response to ABA and water deficit conditions to regulate plant growth [14]. Likewise, high expression levels of Zmhdz7, Zmhdz1, Zmhdz3 and Zmhdz2 of subgroup $\square 1$ were 
induced while the expression of Zmhdz15 was greatly downregulated by drought stress in maize [26]. Besides this, under drought stress, the downregulation of Class I members such as SiHZ25 and SiHZ43 (subgroup $\square$ ) can be linked to an increase in plant water content and malondialdehyde content to enhance drought tolerance in sesame [59]. Thus, it is most likely that SiHD-ZIP Class I genes may function in a coordinated manner, particularly, SiHZ7 and SiHZ35 from subgroup $\square \square \square$ in the developmental reprogramming of sesame at early seedling stage in response to drought stress, as already reported in Arabidopsis [64]. Thus, these results clearly suggest both upregulation and downregulation of SiHD-ZIP Class I gene members under drought stress responses at seedling stage in sesame. However, it is most likely that gene members of SiHD-ZIP Class I belonging to subgroups $\square \square$ and $\square \square$ have more functional importance to regulate early drought stress responses in sesame.

\section{Conclusion}

This study reveals differential gene expression patterns of SiHD-ZIP Class I under drought stress in sesame seedlings. Sesame is known for its sensitivity to drought at early seedling stage. Therefore, this study provides meaningful information to extend our understanding on sesame drought responses at early seedling stage. In the light of results, SiHDZIP Class I members from subgroup $\square \square \square($ SiHZ7 and SiHZ35) can be considered good candidate genes to have significant role in drought stress developmental reprogramming of early growth stages under drought stress in sesame. Thus, the given study may help to reveal the SiHD-ZIP Class I mediated regulatory networks of drought stress response at early seedling stage in sesame. Moreover, these findings may provide baseline molecular information to design new strategies to engineer sesame novel varieties with improved drought tolerance.

\section{Declarations}

This manuscript present original research.

\section{References}

1. Chivenge P, Mabhaudhi T, Modi AT, Mafongoya P (2015) The potential role of neglected and underutilised crop species as future crops under water scarce conditions in Sub-Saharan Africa. International Journal of Environmental Research and Public Health 12:5685-5711. https://doi.org/10.3390/ijerph120605685

2. Wang L, Yu S, Tong C, et al (2014) Genome sequencing of the high oil crop sesame provides insight into oil biosynthesis. Genome Biology 15:R39. https://doi.org/10.1186/gb-2014-15-2-r39

3. Dossa K, Wei X, Li D, et al (2016) Insight into the AP2/ERF transcription factor superfamily in sesame and expression profiling of DREB subfamily under drought stress. BMC Plant Biology 16:171. https://doi.org/10.1186/s12870-016-0859-4 
4. Pathak N, Rai AK, Kumari R, Bhat K V. (2014) Value addition in sesame: A perspective on bioactive components for enhancing utility and profitability. Pharmacognosy Reviews 8:147-155. https://doi.org/10.4103/0973-7847.134249

5. Nadeem A, Kashani S, Ahmed N, et al (2015) Growth and Yield of Sesame ( Sesamum indicum L .) under the Influence of Planting Geometry and Irrigation Regimes. American Journal of Plant Sciences 6:980-986

6. Li D, Jin C, Duan S, et al (2017) MYB89 Transcription Factor Represses Seed Oil Accumulation. Plant Physiology 173:1211-1225. https://doi.org/10.1104/pp.16.01634

7. Dossa K, Li D, Zhou R, et al (2019) The genetic basis of drought tolerance in the high oil crop Sesamum indicum. Plant Biotechnology Journal 17:1788-1803. https://doi.org/10.1111/pbi.13100

8. Shen W, Li H, Teng R, et al (2019) Genomic and transcriptomic analyses of HD-Zip family transcription factors and their responses to abiotic stress in tea plant (Camellia sinensis). Genomics 111:1142-1151. https://doi.org/10.1016/j.ygeno.2018.07.009

9. Mukherjee K, Brocchieri L, Bürglin TR (2009) A comprehensive classification and evolutionary analysis of plant homeobox genes. Molecular Biology and Evolution 26:2775-2794. https://doi.org/10.1093/molbev/msp201

10. Ebrahimian-Motlagh S, Ribone PA, Thirumalaikumar VP, et al (2017) JUNGBRUNNEN1 confers drought tolerance downstream of the HD-Zip I Transcription factor AtHB13. Frontiers in Plant Science 8:1-12. https://doi.org/10.3389/fpls.2017.02118

11. Ding Z, Fu L, Yan Y, et al (2017) Genome-wide characterization and expression profiling of HD-Zip gene family related to abiotic stress in cassava. PLoS ONE 12:1-20. https://doi.org/10.1371/journal.pone.0173043

12. Gough J, Karplus K, Hughey R, Chothia C (2001) Assignment of homology to genome sequences using a library of hidden Markov models that represent all proteins of known structure. Journal of Molecular Biology 313:903-919. https://doi.org/10.1006/jmbi.2001.5080

13. Song A, Li P, Xin J, et al (2016) Transcriptome-wide survey and expression profile analysis of putative chrysanthemum HD-Zip I and II Genes. Genes 7:. https://doi.org/10.3390/genes7050019

14. Henriksson E, Olsson ASB, Johannesson H, et al (2005) Homeodomain leucine zipper class I genes in Arabidopsis. Expression patterns and phylogenetic relationships. Plant Physiology 139:509-518. https://doi.org/10.1104/pp.105.063461

15. Capella M, Ribone PA, Arce AL, Chan RL (2016) Homeodomain-leucine zipper transcription factors: Structural features of these proteins, unique to plants. Elsevier Inc.

16. Palena CM, Gonzalez DH, Chan RL (1999) A monomer-dimer equilibrium modulates the interaction of the sunflower homeodomain leucine-zipper protein Hahb-4 with DNA. Biochemical Journal 341:81-87. https://doi.org/10.1042/0264-6021:3410081

17. Ariel FD, Manavella PA, Dezar CA, Chan RL (2007) The true story of the HD-Zip family. Trends in Plant Science 12:419-426. https://doi.org/10.1016/j.tplants.2007.08.003

18. Mukherjee K, Bürglin TR (2006) MEKHLA, a novel domain with similarity to PAS domains, is fused to plant homeodomain-leucine zipper III proteins. Plant Physiology 140:1142-1150. https://doi.org/10.1104/pp.105.073833

19. Schrick K, Nguyen D, Karlowski WM, Mayer KFX (2004) START lipid/sterol-binding domains are amplified in plants and are predominantly associated with homeodomain transcription factors. Genome biology 5:1-16. https://doi.org/10.1186/gb-2004-5-6-r41 
20. Chai W, Si W, Ji W, et al (2018) Genome-Wide Investigation and Expression Profiling of HD-Zip Transcription Factors in Foxtail Millet (Setaria italica L.). BioMed Research International 2018:18. https://doi.org/10.1155/2018/8457614

21. Zhao Y, Ma Q, Jin X, et al (2014) A novel maize homeodomain-leucine zipper (HD-Zip) i gene, Zmhdz10, positively regulates drought and salt tolerance in both rice and arabidopsis. Plant and Cell Physiology 55:1142-1156. https://doi.org/10.1093/pcp/pcu054

22. Yue H, Shu D, Wang M, et al (2018) Genome-wide identification and expression analysis of the hd-zip gene family in wheat (Triticum aestivum 1.). Genes 9:. https://doi.org/10.3390/genes9020070

23. Söderman E, Hjellström M, Fahleson J, Engström P (1999) The HD-Zip gene ATHB6 in Arabidopsis is expressed in developing leaves, roots and carpels and up-regulated by water deficit conditions. Plant Molecular Biology 40:1073-1083. https://doi.org/10.1023/A:1006267013170

24. Zhao Y, Zhou Y, Jiang H, et al (2011) Systematic analysis of sequences and expression patterns of droughtresponsive members of the HD-Zip gene family in maize. PLoS ONE 6:20-21. https://doi.org/10.1371/journal.pone.0028488

25. Harris JC, Hrmova M, Lopato S, Langridge P (2011) Modulation of plant growth by HD-Zip class I and II transcription factors in response to environmental stimuli. New Phytologist 190:823-837. https://doi.org/10.1111/j.1469-8137.2011.03733.x

26. Zhao Y, Zhou Y, Jiang H, et al (2011) Systematic analysis of sequences and expression patterns of droughtresponsive members of the HD-Zip gene family in maize. PLoS ONE 6:20-21. https://doi.org/10.1371/journal.pone.0028488

27. Wei M, Liu A, Zhang Y, et al (2019) Genome-wide characterization and expression analysis of the HD-Zip gene family in response to drought and salinity stresses in sesame. BMC Genomics 20:1-13. https://doi.org/10.1186/s12864-019-6091-5

28. Zheng Y, Jiao C, Sun H, et al (2016) iTAK: A program for genome-wide prediction and classification of plant transcription factors, transcriptional regulators, and protein kinases. Molecular Plant 9:1667-1670. https://doi.org/10.1016/j.molp.2016.09.014

29. Kumar S, Stecher G, Li M, et al (2018) MEGA X: Molecular evolutionary genetics analysis across computing platforms. Molecular Biology and Evolution 35:1547-1549.

https://doi.org/10.1093/molbev/msy096

30. Huson DH, Scornavacca C (2012) Dendroscope 3: An interactive tool for rooted phylogenetic trees and networks. Systematic Biology 61:1061-1067. https://doi.org/10.1093/sysbio/sys062

31. Bailey TL, Boden M, Buske FA, et al (2009) MEME Suite: Tools for motif discovery and searching. Nucleic Acids Research 37:202-208. https://doi.org/10.1093/nar/gkp335

32. Sigrist CJA, De Castro E, Cerutti L, et al (2013) New and continuing developments at PROSITE. Nucleic Acids Research 41:344-347. https://doi.org/10.1093/nar/gks1067

33. Gasteiger E, Hoogland C, Gattiker A, et al (2005) The Proteomics Protocols Handbook. The Proteomics Protocols Handbook 571-608. https://doi.org/10.1385/1592598900

34. Mitchell AL, Attwood TK, Babbitt PC, et al (2019) InterPro in 2019: Improving coverage, classification and access to protein sequence annotations. Nucleic Acids Research 47:D351-D360. https://doi.org/10.1093/nar/gky1100

35. Letunic I, Bork P (2018) 20 years of the SMART protein domain annotation resource. Nucleic Acids Research 46:D493-D496. https://doi.org/10.1093/nar/gkx922 
36. Hu B, Jin J, Guo AY, et al (2015) GSDS 2.0: An upgraded gene feature visualization server. Bioinformatics 31:1296-1297. https://doi.org/10.1093/bioinformatics/btu817

37. Rio DC, Ares M, Hannon GJ, Nilsen TW (2010) Purification of RNA using TRIzol (TRI Reagent). Cold Spring Harbor Protocols 5:1-4. https://doi.org/10.1101/pdb.prot5439

38. Livak KJ, Schmittgen TD (2001) Analysis of relative gene expression data using real-time quantitative PCR and the 2- $\Delta \Delta \mathrm{CT}$ method. Methods 25:402-408. https://doi.org/10.1006/meth.2001.1262

39. Li W, Dong J, Cao M, et al (2019) Genome-wide identification and characterization of HD-ZIP genes in potato. Gene 697:103-117. https://doi.org/10.1016/j.gene.2019.02.024

40. Agalou A, Purwantomo S, Övernäs E, et al (2008) A genome-wide survey of HD-Zip genes in rice and analysis of drought-responsive family members. Plant Molecular Biology 66:87-103.

https://doi.org/10.1007/s11103-007-9255-7

41. Zhang Z, Chen X, Guan X, et al (2014) A genome-wide survey of homeodomain-leucine zipper genes and analysis of cold-responsive HD-Zip I members' expression in tomato. Bioscience, Biotechnology and Biochemistry 78:1337-1349. https://doi.org/10.1080/09168451.2014.923292

42. Li Z, Gao Z, Li R, et al (2020) Genome-wide identification and expression profiling of HD-ZIP gene family in Medicago truncatula. Genomics 112:3624-3635. https://doi.org/10.1016/j.ygeno.2020.03.008

43. Jiang H, Jin J, Liu H, et al (2015) Genome-wide analysis of HD-Zip genes in grape (Vitis vinifera). Tree Genetics and Genomes 11:. https://doi.org/10.1007/s11295-014-0827-9

44. Chen X, Chen Z, Zhao H, et al (2014) Genome-wide analysis of soybean HD-Zip gene family and expression profiling under salinity and drought treatments. PLoS ONE 9:.

https://doi.org/10.1371/journal.pone.0087156

45. Khan N, Hu C mei, Khan WA, et al (2018) Genome-wide Identification, Classification, and Expression Pattern of Homeobox Gene Family in Brassica rapa under Various Stresses. Scientific Reports 8:. https://doi.org/10.1038/s41598-018-34448-x

46. Hu R, Chi X, Chai G, et al (2012) Genome-Wide identification, evolutionary expansion, and expression profile of Homeodomain-Leucine zipper gene family in poplar (populus trichocarpa). PLoS ONE 7:. https://doi.org/10.1371/journal.pone.0031149

47. Wang H, Lin J, Li XG, Chang Y (2015) Genome-wide identification of pear HD-Zip gene family and expression patterns under stress induced by drought, salinity, and pathogen. Acta Physiologiae Plantarum 37:1-19. https://doi.org/10.1007/s11738-015-1933-5

48. Zhang CH, Ma RJ, Shen ZJ, et al (2014) Genome-wide analysis of the homeodomain-leucine zipper (HDZIP) gene family in peach (Prunus persica). Genetics and Molecular Research 13:2654-2668. https://doi.org/10.4238/2014.April.8.8

49. Li L, Zheng T, Zhuo X, et al (2019) Genome-wide identification, characterization and expression analysis of the HD-Zip gene family in the stem development of the woody plant Prunus mume. PeerJ 2019:. https://doi.org/10.7717/peerj.7499

50. Zhang J, Wu J, Guo M, et al (2020) Genome-wide characterization and expression profiling of Eucalyptus grandis HD-Zip gene family in response to salt and temperature stress. BMC Plant Biology 20:1-15. https://doi.org/10.1186/s12870-020-02677-w

51. Belamkar V, Weeks NT, Bharti AK, et al (2014) Comprehensive characterization and RNA-Seq profiling of the HD-Zip transcription factor family in soybean (Glycine max) during dehydration and salt stress. BMC Genomics 15:1-25. https://doi.org/10.1186/1471-2164-15-950 
52. Zhang H, Miao H, Wang L, et al (2013) Genome sequencing of the important oilseed crop Sesamum indicum L. Genome Biology 14:1-9. https://doi.org/10.1186/gb-2013-14-1-401

53. Ebrahimian-Motlagh S, Ribone PA, Thirumalaikumar VP, et al (2017) JUNGBRUNNEN1 confers drought tolerance downstream of the HD-Zip I Transcription factor AtHB13. Frontiers in Plant Science 8:1-12. https://doi.org/10.3389/fpls.2017.02118

54. Song A, Li P, Xin J, et al (2016) Transcriptome-wide survey and expression profile analysis of putative chrysanthemum HD-Zip I and II Genes. Genes 7:. https://doi.org/10.3390/genes7050019

55. Cao J, Xiong L (2014) Protein sequence classification with improved extreme learning machine algorithms. BioMed Research International 2014:12. https://doi.org/10.1155/2014/103054

56. Lopes Cardoso D, Sharpe C (2017) Relating protein functional diversity to cell type number identifies genes that determine dynamic aspects of chromatin organisation as potential contributors to organismal complexity. PLoS ONE 12:1-16. https://doi.org/10.1371/journal.pone.0185409

57. Boeckmann B, Blatter MC, Famiglietti L, et al (2005) Protein variety and functional diversity: Swiss-Prot annotation in its biological context. Comptes Rendus - Biologies 328:882-899. https://doi.org/10.1016/j.crvi.2005.06.001

58. Dezar CA, Fedrigo G V., Chan RL (2005) The promoter of the sunflower HD-Zip protein gene Hahb4 directs tissue-specific expression and is inducible by water stress, high salt concentrations and ABA. Plant Science 169:447-456. https://doi.org/10.1016/j.plantsci.2005.04.008

59. Li Y, Bai B, Wen F, et al (2019) Genome-wide identification and expression analysis of HD-ZIP I gene subfamily in Nicotiana tabacum. Genes 10:1-19. https://doi.org/10.3390/genes10080575

60. Son O, Hur YS, Kim YK, et al (2010) ATHB12, an ABA-inducible homeodomain-leucine zipper (HD-Zip) protein of arabidopsis, negatively regulates the growth of the inflorescence stem by decreasing the expression of a gibberellin 20-oxidase gene. Plant and Cell Physiology 51:1537-1547. https://doi.org/10.1093/pcp/pcq108

61. Olsson ASB, Engström P, Söderman E (2004) The homeobox genes ATHB12 and ATHB7 encode potential regulators of growth in response to water deficit in Arabidopsis. Plant Molecular Biology 55:663-677. https://doi.org/10.1007/s11103-004-1581-4

62. Ré DA, Capella M, Bonaventure G, Chan RL (2014) Arabidopsis AtHB7 and AtHB12 evolved divergently to fine tune processes associated with growth and responses to water stress. BMC Plant Biology 14:1-14. https://doi.org/10.1186/1471-2229-14-150

63. Cabello J V., Chan RL (2012) The homologous homeodomain-leucine zipper transcription factors HaHB1 and AtHB13 confer tolerance to drought and salinity stresses via the induction of proteins that stabilize membranes. Plant Biotechnology Journal 10:815-825. https://doi.org/10.1111/j.1467-7652.2012.00701.x

64. Ré DA, Capella M, Bonaventure G, Chan RL (2014) Arabidopsis AtHB7 and AtHB12 evolved divergently to fine tune processes associated with growth and responses to water stress. BMC Plant Biology 14:1-14. https://doi.org/10.1186/1471-2229-14-150 
Table 1 List of HD-ZIP proteins classified in sesame: their amino acid sequence length (a.a), theoratical iso-electric point $(\mathrm{pI}) /$ molecular weight $(\mathrm{MW}, \mathrm{kDa})$ and their gene distribution on different linkage groups (LGs)

\begin{tabular}{|c|c|c|c|c|c|c|c|}
\hline $\begin{array}{l}\text { SiHDZI } \\
\text { P name }\end{array}$ & $\begin{array}{c}\text { Protein } \\
\text { accession id }\end{array}$ & $\begin{array}{c}\text { Amino } \\
\text { acid } \\
\text { sequence } \\
\text { length } \\
\text { (a.a) } \\
\end{array}$ & $\begin{array}{c}\text { Theoratical } \\
\text { pI/MW(kDa } \\
\text { ) }\end{array}$ & $\begin{array}{c}\text { Gene symbol } \\
\text { id }\end{array}$ & $\begin{array}{l}\text { Linkag } \\
\text { e group } \\
\text { (LG) }\end{array}$ & $\begin{array}{c}\text { Exon } \\
\text { coun } \\
t\end{array}$ & $\begin{array}{c}\text { Clas } \\
\mathrm{s}\end{array}$ \\
\hline SiHZ1 & $\begin{array}{c}\text { XP_011075631. } \\
1\end{array}$ & 221 & $6.02 / 24.8$ & $\begin{array}{c}\text { LOC10516006 } \\
1\end{array}$ & LG1 & 4 & $\overline{\text { II }}$ \\
\hline SiHZ2 & XP_011080516. & 845 & $5.96 / 92.4$ & $\begin{array}{c}\text { LOC } 10516375 \\
7\end{array}$ & LG1 & 18 & III \\
\hline SiHZ3a & XP_011087051. & 364 & $4.99 / 40.1$ & $\begin{array}{c}\text { LOC } 10516861 \\
2\end{array}$ & LG1 & 5 & II \\
\hline SiHZ3b & XP_011087055. & 363 & $4.99 / 40$ & $\begin{array}{c}\text { LOC10516861 } \\
2\end{array}$ & LG1 & 5 & II \\
\hline SiHZ4 & XP_011089804. & 304 & $8.46 / 34$ & $\begin{array}{c}\text { LOC } 10517064 \\
6\end{array}$ & LG1 & 4 & II \\
\hline SiHZ5 & $\begin{array}{c}\text { XP_011091721. } \\
2\end{array}$ & 837 & $6.47 / 91.7$ & $\begin{array}{c}\text { LOC } 10517207 \\
8\end{array}$ & LG1 & 9 & IV \\
\hline SiHZ6a & XP_011070327. & 817 & $5.40 / 90.6$ & $\begin{array}{c}\text { LOC } 10515601 \\
2\end{array}$ & LG2 & 13 & IV \\
\hline SiHZ6b & $\begin{array}{c}\text { XP_011070328. } \\
1\end{array}$ & 817 & $5.40 / 90.6$ & $\begin{array}{c}\text { LOC } 10515601 \\
2\end{array}$ & LG2 & 13 & IV \\
\hline SiHZ6c & $\begin{array}{c}\text { XP_011070329. } \\
1\end{array}$ & 816 & $5.40 / 90.5$ & $\begin{array}{c}\text { LOC10515601 } \\
2\end{array}$ & LG2 & 13 & IV \\
\hline SiHZ7 & XP_011070385. & 201 & $7.71 / 23$ & $\begin{array}{c}\text { LOC } 10515605 \\
1\end{array}$ & LG2 & 2 & I \\
\hline SiHZ8 & XP_011070725. & 212 & $8.74 / 24$ & $\begin{array}{c}\text { LOC } 10515632 \\
7\end{array}$ & LG2 & 4 & II \\
\hline SiHZ9 & $\begin{array}{c}\text { XP_011071017. } \\
1\end{array}$ & 292 & $5.96 / 33.2$ & $\begin{array}{c}\text { LOC } 10515655 \\
1\end{array}$ & LG2 & 3 & I \\
\hline SiHZ10 & $\begin{array}{c}\text { XP_011071034. } \\
1\end{array}$ & 316 & $4.73 / 36$ & $\begin{array}{c}\text { LOC } 10515656 \\
4\end{array}$ & LG2 & 3 & I \\
\hline SiHZ11 & XP_011072039. & 717 & $6.30 / 79.1$ & $\begin{array}{c}\text { LOC } 10515733 \\
9\end{array}$ & LG3 & 10 & IV \\
\hline SiHZ12a & XP_011072373. & 757 & $6.50 / 84.2$ & $\begin{array}{c}\text { LOC } 10515764 \\
7\end{array}$ & LG3 & 11 & IV \\
\hline SiHZ12b & XP_020548412. & 659 & $8.50 / 73.9$ & $\begin{array}{c}\text { LOC } 10515764 \\
7\end{array}$ & LG3 & 11 & IV \\
\hline SiHZ13 & XP_011072422. & 840 & $6.28 / 92.3$ & $\begin{array}{c}\text { LOC } 10515767 \\
8\end{array}$ & LG3 & 18 & III \\
\hline SiHZ14a & $\begin{array}{c}\text { XP_011073660. } \\
1\end{array}$ & 269 & $8.71 / 29.9$ & $\begin{array}{c}\text { LOC } 10515856 \\
9\end{array}$ & LG3 & 4 & II \\
\hline SiHZ14b & XP_011073661. & 242 & $8.63 / 26.8$ & $\begin{array}{c}\text { LOC } 10515856 \\
9\end{array}$ & LG3 & 4 & II \\
\hline SiHZ15 & $\begin{array}{c}\text { XP_011075258. } \\
1\end{array}$ & 325 & $7.58 / 36.2$ & $\begin{array}{c}\text { LOC } 10515977 \\
0\end{array}$ & LG4 & 4 & II \\
\hline SiHZ16 & $\begin{array}{c}\text { XP_011075470. } \\
1\end{array}$ & 307 & $6.05 / 34.8$ & $\begin{array}{c}\text { LOC } 10515993 \\
9\end{array}$ & LG4 & 3 & I \\
\hline SiHZ17 & XP_020549042. & 220 & $5.21 / 25.9$ & $\begin{array}{c}\text { LOC } 10516029 \\
2\end{array}$ & LG4 & 2 & I \\
\hline SiHZ18 & XP_011076208. & 811 & $6.48 / 88.5$ & $\begin{array}{c}\text { LOC } 10516050 \\
6\end{array}$ & LG4 & 9 & IV \\
\hline
\end{tabular}


SiHZ19a XP_011076980. 836

SiHZ19b XP_011076981. 835

SiHZ19c XP_011076983. 833

SiHZ20 XP_011081291. 307

SiHZ21 XP_011081776. 841

SiHZ22a $\quad$ XP_011084330. 831

SiHZ22b XP_011084331. 805

$\begin{array}{lll} & 1 \\ \text { SiHZ22c } & \text { XP_011084333. } & 803\end{array}$

STH220 XP_011084333. 1

SiHZ23 XP_011085207. 847

$\begin{array}{ccc} & 1 \\ \text { SiHZ24a } & \text { XP_020552078. } & 696\end{array}$

SiHZ24b XP_020552079. 691

$\begin{array}{ccc} & 1 \\ \text { SiHZ24c } & \text { XP_020552080. } & 694\end{array}$

SiHZ25 XP_020551655. 273

SiHZ26 XP_011085824. 259

SiHZ26 XP_011085824.

SiHZ27 XP_020551334. 660

SiHZ28 XP_011085930. 698

SiHZ29 XP_011086174. 733

SiHZ29 XP_01108617.
2

SiHZ30 XP_011086401. 340

SiHZ31 XP_011086973. 727

SiHZ32 XP_011087214. 293

SiHZ33 XP_011089294. 838

SiHZ34 XP_011089912. 839

SiHZ35 XP_011093009. 230

SiHZ36a XP_011093166. 835

SiHZ36b XP_011093167. 834

SiHZ37 XP_011093277. 315

SiHZ38 XP_011093390. 373

SiHZ40a XP_011096101. 726

$\begin{array}{lllll}5.93 / 91.8 & \text { LOC } 10516109 & \text { LG4 } & 18 & \text { III }\end{array}$

LOC10516109 LG4 $18 \quad$ III

$\begin{array}{lc}5.86 / 91.7 & \text { LOC105 } \\ 9 & \end{array}$

5.93/91.5 LOC10516109 LG4 $18 \quad$ III

8.51/34.1 LOC10516435 LG6 4 II

6.00/92.8 LOC10516473 LG6 $19 \quad$ III

5.99/91.8 LOC10516661 LG7 $18 \quad$ III

6.08/88.6 LOC10516661 LG7 $18 \quad$ III

$\begin{array}{lllll}6.05 / 88.6 & \text { LOC10516661 } & \text { LG7 } & 18 & \text { III }\end{array}$

6.18/93.1 LOC10516726 LG8 18 III

6.22/77.2 LOC10516736 LG8 $11 \quad$ IV

$\begin{array}{lllll}\text { 6.31/76.7 LOC10516736 LG8 } & \text { L } & \text { IV }\end{array}$

6.14/77 LOC10516736 LG8 $11 \quad$ IV

6.51/31.1 LOC10516766 LG8 $3 \quad$ I

$\begin{array}{lllll}4.84 / 29.9 & \text { LOC10516770 } & \text { LG8 } & 4 & \text { I }\end{array}$

6.59/74.3 LOC10516781 LG8 $11 \quad$ IV

6.63/78.8 LOC10516781 LG8 $11 \quad$ IV

5.83/81 LOC10516797 LG8 $13 \quad$ IV

5.34/38.4 LOC10516814 LG8 3 I

5.95/79.9 LOC10516854 LG8 $10 \quad$ IV

$\begin{array}{lllll}5.04 / 33.4 & \text { LOC10516876 } & \text { LG8 } & 3 & \text { I }\end{array}$

$\begin{array}{lllll}6.00 / 91.8 & \text { LOC10516876 } & \text { LG9 } & 18 & \text { III }\end{array}$

$\begin{array}{lllll}5.81 / 91.9 & \text { LOC10517072 LG9 } & 18 & \text { III }\end{array}$

4.97/26.5 LOC10517306 LG11 2 I

5.86/91.2 LOC10517318 LG11 9 IV

5.86/91.1 LOC10517318 LG11 9

8.64/35.4 LOC10517328 LG11 4 II

5.89/41.1 LOC10517337 LG11 4 II

5.66/79.6 LOC10517537 LG12 $13 \quad$ IV 


\begin{tabular}{|c|c|c|c|c|c|c|c|}
\hline SiHZ40b & $\begin{array}{c}\text { XP_011096102. } \\
1\end{array}$ & 726 & $5.66 / 79.6$ & $\begin{array}{c}\text { LOC } 10517537 \\
2\end{array}$ & LG12 & 13 & IV \\
\hline SiHZ40c & $\begin{array}{c}\text { XP_011096103. } \\
1\end{array}$ & 726 & $5.66 / 79.6$ & $\begin{array}{c}\text { LOC } 10517537 \\
2\end{array}$ & LG12 & 13 & IV \\
\hline SiHZ40d & XP_020554107. & 726 & $5.66 / 79.6$ & $\begin{array}{c}\text { LOC } 10517537 \\
2\end{array}$ & LG12 & 13 & IV \\
\hline SiHZ41a & $\begin{array}{c}\text { XP_011096678. } \\
1\end{array}$ & 852 & $5.85 / 93.4$ & $\begin{array}{c}\text { LOC } 10517578 \\
8\end{array}$ & LG12 & 18 & III \\
\hline SiHZ41b & $\begin{array}{c}\text { XP_020554026. } \\
1\end{array}$ & 853 & $5.85 / 93.5$ & $\begin{array}{c}\text { LOC } 10517578 \\
8\end{array}$ & LG12 & 18 & III \\
\hline SiHZ41c & $\begin{array}{c}\text { XP_020554106. } \\
1\end{array}$ & 726 & $5.66 / 79.6$ & $\begin{array}{c}\text { LOC10517578 } \\
8\end{array}$ & LG12 & 18 & IV \\
\hline SiHZ42 & $\begin{array}{c}\text { XP_011097532. } \\
1\end{array}$ & 305 & $4.92 / 34.4$ & $\begin{array}{c}\text { LOC } 10517643 \\
3\end{array}$ & LG13 & 3 & I \\
\hline SiHZ43 & XP_011098601. & 281 & $6.08 / 32.2$ & $\begin{array}{c}\text { LOC } 10517723 \\
0\end{array}$ & LG15 & 3 & I \\
\hline SiHZ44 & $\begin{array}{c}\text { XP_011099420. } \\
1\end{array}$ & 269 & $8.09 / 29.9$ & $\begin{array}{c}\text { LOC10517785 } \\
2\end{array}$ & LG15 & 3 & II \\
\hline SiHZ45 & $\begin{array}{c}\text { XP_011100030. } \\
1\end{array}$ & 328 & $4.73 / 36.9$ & $\begin{array}{c}\text { LOC10517828 } \\
0\end{array}$ & LG15 & 3 & I \\
\hline
\end{tabular}

Supplementary Table S1 List of identified HD-ZIP TFs in sesame by protein sequence homology (A. thaliana) and domain(s) assignment by HMM (Hidden Markov's Model)

\begin{tabular}{|c|c|c|c|c|c|}
\hline Protein ID & Gene symbol & $\begin{array}{c}\text { Linkage } \\
\text { group } \\
\text { (LG) }\end{array}$ & Chromosomal location & $\begin{array}{l}\text { Exon } \\
\text { count }\end{array}$ & $\begin{array}{c}\text { SiHD-ZIP } \\
\text { assigned } \\
\text { name }\end{array}$ \\
\hline 1 XP_011075631.1 & LOC105160061 & LG1 & $9307691 . .9310369$ & 4 & SiHZ1 \\
\hline 2 XP_011080516.1 & LOC105163757 & LG1 & $\begin{array}{c}\text { 11862772..11869357, } \\
\text { complement }\end{array}$ & 18 & $\mathrm{SiHZ2}$ \\
\hline 3 XP_011087051.1 & LOC105168612 & LG1 & $15178310 . .15180598$ & 5 & $\mathrm{SiHZ3a}$ \\
\hline 4 XP_011087055.1 & LOC105168612 & LG1 & $15178310 . .15180598$ & 5 & $\mathrm{SiHZ3b}$ \\
\hline 5 XP_011089804.1 & LOC105170646 & LG1 & $\begin{array}{c}\text { 16504182..16506768, } \\
\text { complement }\end{array}$ & 4 & $\mathrm{SiHZ4}$ \\
\hline 6 XP_011091721.2 & LOC 105172078 & LG1 & $17471921 . .17477926$ & 9 & $\mathrm{SiHZ5}$ \\
\hline 7 XP_011070327.1 & LOC105156012 & LG2 & $12848062 . .12854969$ & 13 & SiHZ6a \\
\hline 8 XP_011070328.1 & LOC105156012 & LG2 & $12848062 . .12854969$ & 13 & $\mathrm{SiHZ6b}$ \\
\hline 9 XP_011070329.1 & LOC105156012 & LG2 & $12848062 . .12854969$ & 13 & $\mathrm{SiHZ6c}$ \\
\hline 1 XP_011070385.1 & LOC 105156051 & LG2 & $\begin{array}{c}\text { 13373296..13374667, } \\
\text { complement }\end{array}$ & 2 & SiHZ7 \\
\hline 1 XP_011070725.1 & LOC105156327 & LG2 & $15262273 . .15264616$ & 4 & SiHZ8 \\
\hline 1 XP_011071017.1 & LOC105156551 & LG2 & $17326495 . .17328901$ & 3 & SiHZ9 \\
\hline 1 XP_011071034.1 & LOC105156564 & LG2 & $17420046 . .17422085$ & 3 & SiHZ10 \\
\hline 1 XP_011072039.1 & LOC105157339 & LG3 & $5902722 . .5906521$ & 10 & SiHZ11 \\
\hline 1 XP_011072373.1 & LOC105157647 & LG3 & $\begin{array}{c}8060164 . .8068299 \\
\text { complement }\end{array}$ & 11 & SiHZ12a \\
\hline 1 XP_020548412.1 & LOC105157647 & LG3 & $\begin{array}{c}8060164 . .8068299 \\
\text { complement }\end{array}$ & 11 & SiHZ12b \\
\hline
\end{tabular}


$8290214 . .8296829$

1 XP_011073660.1 LOC105158569 LG3

16324701..16326391,

SiHZ14a

1 XP_011073661.1 LOC105158569 LG3

complement

2 XP_011075258.1 LOC105159770 LG4

16324701..16326391,

SiHZ14b complement

2 XP_011075470.1 LOC105159939 LG4

365616..367287, complement

SiHZ15

2 XP_020549042.1 LOC105160292 LG4

2 XP_011076208.1 LOC105160506 LG4

2 XP_011076980.1 LOC105161099 LG4

2 XP_011076981.1 LOC105161099 LG4

2 XP_011076983.1 LOC105161099 LG4

2 XP_011081291.1 LOC105164355 LG6

2 XP_011081776.1 LOC105164733 LG6

2 XP_011084330.1 LOC105166610 LG7

3 XP_011084331.1 LOC105166610 LG7

3 XP_011084333.1 LOC105166610 LG7

3 XP_011085207.1 LOC105167264 LG8

3 XP_020552078.1 LOC105167361 LG8

3 XP_020552079.1 LOC105167361 LG8

3 XP_020552080.1 LOC105167361 LG8

3 XP_020551655.1 LOC105167666 LG8

3 XP_011085824.1 LOC105167706 LG8

3 XP_020551334.1 LOC105167814 LG8

3 XP_011085930.1 LOC105167814 LG8

4 XP_011086174.2 LOC105167974 LG8

4 XP_011086401.2 LOC105168148 LG8

4 XP_011086973.1 LOC105168541 LG8

4 XP_011087214.1 LOC105168765 LG8

4 XP_011089294.1 LOC105168765 LG9

4 XP_011089912.1 LOC105170721 LG9

4 XP_011093009.1 LOC105173060 LG11

4 XP_011093166.1 LOC105173189 LG11

3530547..3532360, complement $8756816 . .8758064$

10138345..10143621, complement

15015420..15028495, complement

15015420..15028495, 18 complement

15015420..15028495, $\quad 18 \quad$ SiHZ19c complement

11199920..11201843

17013344..17019333, complement

8239690..8255794, complement

8239690..8255794, complement

8239690..8255794, complement

273987..280075

1066413..1076252, complement

1066413..1076252, complement

1066413..1076252, complement

3612903..3614911

3921244..3924299, complement

6769979..6780673, complement 6769979..6780673,
complement

8640252..8645949

10039939..10041760

13955207..13959698, complement

14739116..14742059, complement

2170825..2178059, complement 5519865..5528848

NC_026155.1 (194980..196348) 1460439..1466477, complement
SiHZ16

SiHZ17

SiHZ18

18 SiHZ19a

SiHZ19b

$\begin{array}{cc}4 & \text { SiHZ20 } \\ 19 & \text { SiHZ21 }\end{array}$

18 SiHZ22a

$18 \quad$ SiHZ22b

$18 \quad$ SiHZ22c

18 SiHZ23

11 SiHZ24a

$11 \quad$ SiHZ24b

$11 \quad \mathrm{SiHZ24c}$

3 SiHZ25

4 SiHZ26

$11 \quad$ SiHZ27

$11 \quad$ SiHZ28

$13 \quad$ SiHZ29

3 SiHZ30

10 SiHZ31

$3 \quad$ SiHZ32

$18 \quad$ SiHZ33

$18 \quad$ SiHZ34

$2 \quad$ SiHZ35

9 SiHZ36a 
4 XP_011093167.1 LOC105173189 LG11

4 XP_011093277.2 LOC105173280 LG11

5 XP_011093390.1 LOC105173376 LG11

5 XP_011096101.1 LOC105175372 LG12

5 XP_011096102.1 LOC105175372 LG12

5 XP_011096103.1 LOC105175372 LG12

5 XP_020554107.1 LOC105175372 LG12

5 XP_011096678.1 LOC105175788 LG12

5 XP_020554026.1 LOC105175788 LG12

5 XP_020554106.1 LOC105175788 LG12

5 XP_011097532.1 LOC105176433 LG13

5 XP_011098601.1 LOC105177230 LG15

6 XP_011099420.1 LOC105177852 LG15

6 XP_011100030.1 LOC105178280 LG15
1460439..1466477, complement

2728727..2731873, complement

5679695..5681389, complement

3439532..3445476, complement

3439532..3445476, complement

3439532..3445476, complement

3439532..3445476, complement 5556645..5562726, complement

5556645..5562726, complement 5556645..5562726, complement 4332486..4335004 820457..822331, complement 4439230..4441002 $9118552 . .9121545$

\begin{tabular}{cc}
9 & SiHZ36b \\
4 & SiHZ37 \\
4 & SiHZ38 \\
13 & SiHZ40a \\
13 & SiHZ40b \\
13 & SiHZ40c \\
13 & SiHZ40d \\
18 & SiHZ41a \\
18 & SiHZ41b \\
18 & SiHZ41c \\
3 & SiHZ42 \\
3 & SiHZ43 \\
3 & SiHZ44 \\
3 & SiHZ45 \\
\hline
\end{tabular}


Supplementary Table S2 Prediction of conserved domains (amino acid sequences) and their function(s) in SiHD-

\begin{tabular}{|c|c|c|c|c|}
\hline Motif \# & Conserved amino acid sequences & Width & $\begin{array}{l}\text { Expasy Prosite } \\
\text { predicted domain }\end{array}$ & $\begin{array}{l}\text { InterPro predicted } \\
\text { domain/Molecular function }\end{array}$ \\
\hline 1. & $\begin{array}{l}\text { KKKRYHRHTPRQIQEMEAFFKECP } \\
\text { HPDDKQRLELSRELGLEPRQVKFW } \\
\text { FQNRRTQMKAQHERHENSQLRAE } \\
\text { NEKLRAENIR } \\
\text { YREALRNAICPNCGGPA }\end{array}$ & 98 & Homeobox & $\begin{array}{l}\text { Homeobox-leucine zipper } \\
\text { protein/DNA binding site }\end{array}$ \\
\hline 2. & $\begin{array}{l}\text { LGJQPRQVKVWFQNRRAREKQKQ } \\
\text { EEVDLZ }\end{array}$ & 29 & Unidentified & None/DNA binding \\
\hline 3. & $\begin{array}{l}\text { PGQPPGJVLSAATSFWLPVPPKRVF } \\
\text { DFLRDENSRSZWDILSNGGPVQEM } \\
\text { AHIANGRDPGNCVSLLRA }\end{array}$ & 67 & Unidentified & $\begin{array}{l}\text { Homeobox-leucine zipper } \\
\text { protein }\end{array}$ \\
\hline 4. & $\begin{array}{l}\text { SKVTWVEHVELEASSVHEVYRPL } \\
\text { YESGLAFGAKRWVAALDRQCZRL } \\
\text { AETM }\end{array}$ & 50 & START & $\begin{array}{l}\text { Homeobox-leucine zipper } \\
\text { protein/Lipid binding }\end{array}$ \\
\hline 5. & $\begin{array}{l}\text { PGNNNGAJZLMYAZLQVPSPLVPT } \\
\text { REFYFLRYCKQHEDGSW }\end{array}$ & 41 & Unidentified & $\begin{array}{l}\text { Homeobox-leucine zipper } \\
\text { protein/Lipid binding }\end{array}$ \\
\hline 6. & $\begin{array}{l}\text { ALPVFTFANQAGLDMLETTLVALQ } \\
\text { DJTLEKILDGSGRKSLFSEFPTIMQQ } \\
\text { GFAILPGGICLSSMGRPVSYEQAVV } \\
\text { WKVLNE DGSVHC }\end{array}$ & 87 & Unidentified & None \\
\hline 7. & $\begin{array}{l}\text { KGEASRESGVVIMNPIKLVEILMD } \\
\text { VNKWSRVFRCIVSRAKT }\end{array}$ & 41 & Unidentified & None \\
\hline 8. & $\begin{array}{l}\text { GEGRKSLLKLAZRMVRGFCDAVN } \\
\text { GSTDDGWTTLSGDGADDV }\end{array}$ & 41 & Unidentified & $\begin{array}{l}\text { Homeobox-leucine zipper } \\
\text { protein/No Go term }\end{array}$ \\
\hline 9. & $\begin{array}{l}\text { QSNMLILQESCTDSTGSYVVYAPV } \\
\text { DIVAMNVVLSGGDPSYIALLPSGF } \\
\text { AILPD }\end{array}$ & 53 & Unidentified & $\begin{array}{l}\text { Homeobox-leucine zipper } \\
\text { protein/No Go term }\end{array}$ \\
\hline 10. & $\begin{array}{l}\text { RKKLRLTKEQVEALEKSFEECNKL } \\
\text { NPKRK }\end{array}$ & 29 & Unidentified & None \\
\hline 11. & $\begin{array}{l}\text { KPMIJELAVAAMDELIRMAQTGEP } \\
\text { LWIPSTDNGAEVLNEEEYVRTFPR } \\
\text { GI }\end{array}$ & 50 & Unidentified & $\begin{array}{l}\text { Homeobox-leucine zipper } \\
\text { protein/Lipid binding }\end{array}$ \\
\hline 12. & ZSLRAENARLKEELDRJKALV & 21 & Unidentified & None \\
\hline 13. & $\begin{array}{l}\text { DJYLLQLCSGMDETGVGACSELIF } \\
\text { APIDRTFPBDAPLLPSGFRIIPLDSN } \\
\text { TGESTAS }\end{array}$ & 57 & Unidentified & None \\
\hline 14. & $\begin{array}{l}\text { LKPLPGSPEAVTLARWICQSYRSH } \\
\text { LGVELLGABCESTDSVLKQJWHHQ } \\
\text { DAILCCSFK }\end{array}$ & 57 & Unidentified & None \\
\hline 15. & $\begin{array}{l}\text { SVJCAKASMLLQNVPPALLVRFLR } \\
\text { EHRSEWADYNVDDYSAAFLKAAP } \\
\text { YAYPGPRIGNLSGTPV }\end{array}$ & 63 & Unidentified & None \\
\hline 16. & $\begin{array}{l}\text { AASPRSLLTJAFZFPFEFLLKATVT } \\
\text { AVARQYVRSVKSSVQS }\end{array}$ & 41 & Unidentified & None \\
\hline 17. & ASFVRCRRLPSGCLIQDMPNG & 21 & Unidentified & None/lipid binding \\
\hline 18. & $\begin{array}{l}\text { DMGHKTPENEMDLIRDDEYESKS } \\
\text { GSDIMEAPSGDDQDPNQR }\end{array}$ & 41 & Unidentified & None \\
\hline 19. & $\begin{array}{l}\text { ANGAPZVGSSGSLLTVAFQILVDSS } \\
\text { PTAK }\end{array}$ & 29 & Unidentified & None \\
\hline 20. & QLPATTLTMCPSCERVATTT & 20 & Unidentified & None \\
\hline
\end{tabular}

ZIP proteins (Class I-IV) 
1

2

3

4

5

6

8

9

10

11

12

13

14

15

16

17

18

19

20

21

22

23

24

25

26

27

28

29

30

31

32

33

34

35

36

37

38

39

40

41

42

43

44

45

46

47

48

49

50

51

52

53

54

55

56

57

58

59

60

61

62

63 
Supplementary Table S3 List of primers of SiHD-ZIP Class I genes

\begin{tabular}{|c|c|c|c|c|}
\hline $\begin{array}{c}\text { SiHD-ZIP } \\
\text { Class I } \\
\text { member } \\
\text { genes }\end{array}$ & mRNA id & Forward primer & Reverse primer & $\begin{array}{l}\text { Product } \\
\text { length } \\
\text { (bp) }\end{array}$ \\
\hline $\mathrm{SiHZ7}$ & $\begin{array}{l}\text { XM_01107208 } \\
3.2\end{array}$ & $\begin{array}{l}\text { TCTAAGCAAATCGAGCAA } \\
\text { GAG }\end{array}$ & TCTGTCTACCTGGGTGAGC & 114 \\
\hline SiHZ9 & $\begin{array}{l}\text { XM_01107271 } \\
5.2\end{array}$ & $\begin{array}{l}\text { CGATGGAAGACTAAGCAG } \\
\text { TT }\end{array}$ & $\begin{array}{l}\text { CTTCAGTGCCATTATCTCG } \\
\text { G }\end{array}$ & 129 \\
\hline SiHZ10 & $\begin{array}{l}\text { XM_01107273 } \\
2.2\end{array}$ & $\begin{array}{l}\text { GTGAAAGCTATGGAGGGTT } \\
\text { G }\end{array}$ & $\begin{array}{l}\text { CAGAGCCTGAAACATGAA } \\
\text { AAG }\end{array}$ & 148 \\
\hline SiHZ16 & $\begin{array}{l}\text { XM_01107716 } \\
8.2\end{array}$ & $\begin{array}{l}\text { TTCATGCTGAGATATTGGC } \\
\text { AC }\end{array}$ & $\begin{array}{l}\text { CTGGAGTCGTCGTTGAAAT } \\
\text { A }\end{array}$ & 138 \\
\hline SiHZ17 & $\begin{array}{l}\text { XM_02069338 } \\
3.1\end{array}$ & $\begin{array}{l}\text { TAAGAGTAGGGCTTTTCCA } \\
\text { CA }\end{array}$ & $\begin{array}{l}\text { ACTGTTTCCTCATCGTCTG } \\
\mathrm{T}\end{array}$ & 165 \\
\hline SiHZ25 & $\begin{array}{l}\text { XM_02069599 } \\
6.1\end{array}$ & $\begin{array}{l}\text { GCTCCTCAAGACTTAAACG } \\
\text { ATGT }\end{array}$ & $\begin{array}{l}\text { TTCAAAGCTCCTCTCCAGG } \\
\text { G }\end{array}$ & 186 \\
\hline SiHZ26 & $\begin{array}{l}\text { XM_01108752 } \\
2.2\end{array}$ & $\begin{array}{l}\text { ATTGTGGTTGAGTGAGGGT } \\
\mathrm{T}\end{array}$ & $\begin{array}{l}\text { TACTTCCACCTCGAAAAAT } \\
\text { CG }\end{array}$ & 148 \\
\hline SiHZ30 & $\begin{array}{l}\text { XM_01108809 } \\
9.2\end{array}$ & $\begin{array}{l}\text { TGTTCCTCTGAGAATTTCG } \\
\text { CC }\end{array}$ & $\begin{array}{l}\text { GATGCAGAGGCATGAAAA } \\
\text { GAAG }\end{array}$ & 181 \\
\hline SiHZ32 & $\begin{array}{l}\text { XM_01108891 } \\
2.2\end{array}$ & $\begin{array}{l}\text { GCTGGAAACAGATTACGA } \\
\text { CA }\end{array}$ & $\begin{array}{l}\text { CTTTTAGACGAAGAACCTC } \\
\text { CG }\end{array}$ & 113 \\
\hline SiHZ35 & $\begin{array}{l}\text { XM_01109470 } \\
7.2\end{array}$ & $\begin{array}{l}\text { CTTGGCTTCCCAGGTTGAA } \\
\text { G }\end{array}$ & $\begin{array}{l}\text { ATCCCCTTCCGGGCTATTT } \\
\mathrm{C}\end{array}$ & 126 \\
\hline SiHZ42 & $\begin{array}{l}\text { XM_01109923 } \\
0.2\end{array}$ & $\begin{array}{l}\text { GAGAGGGATTATGGGGTTC } \\
\mathrm{T}\end{array}$ & $\begin{array}{l}\text { GCTCTCCAATCTCTTTGAG } \\
\mathrm{T}\end{array}$ & 103 \\
\hline SiHZ43 & $\begin{array}{l}\text { XM_01110029 } \\
9.2\end{array}$ & $\begin{array}{l}\text { TCAAACACAGTTATTGGCC } \\
\text { C }\end{array}$ & $\begin{array}{l}\text { GATGTTGTGTTGAGGTTGA } \\
\text { C }\end{array}$ & 132 \\
\hline SiHZ45 & $\begin{array}{l}\text { XM_01110172 } \\
8.2\end{array}$ & $\begin{array}{l}\text { TCTACAGATGAAAACAGCC } \\
\text { C }\end{array}$ & $\begin{array}{l}\text { CTCСТCTTCTTCTCCGAAA } \\
\text { C }\end{array}$ & 137 \\
\hline
\end{tabular}




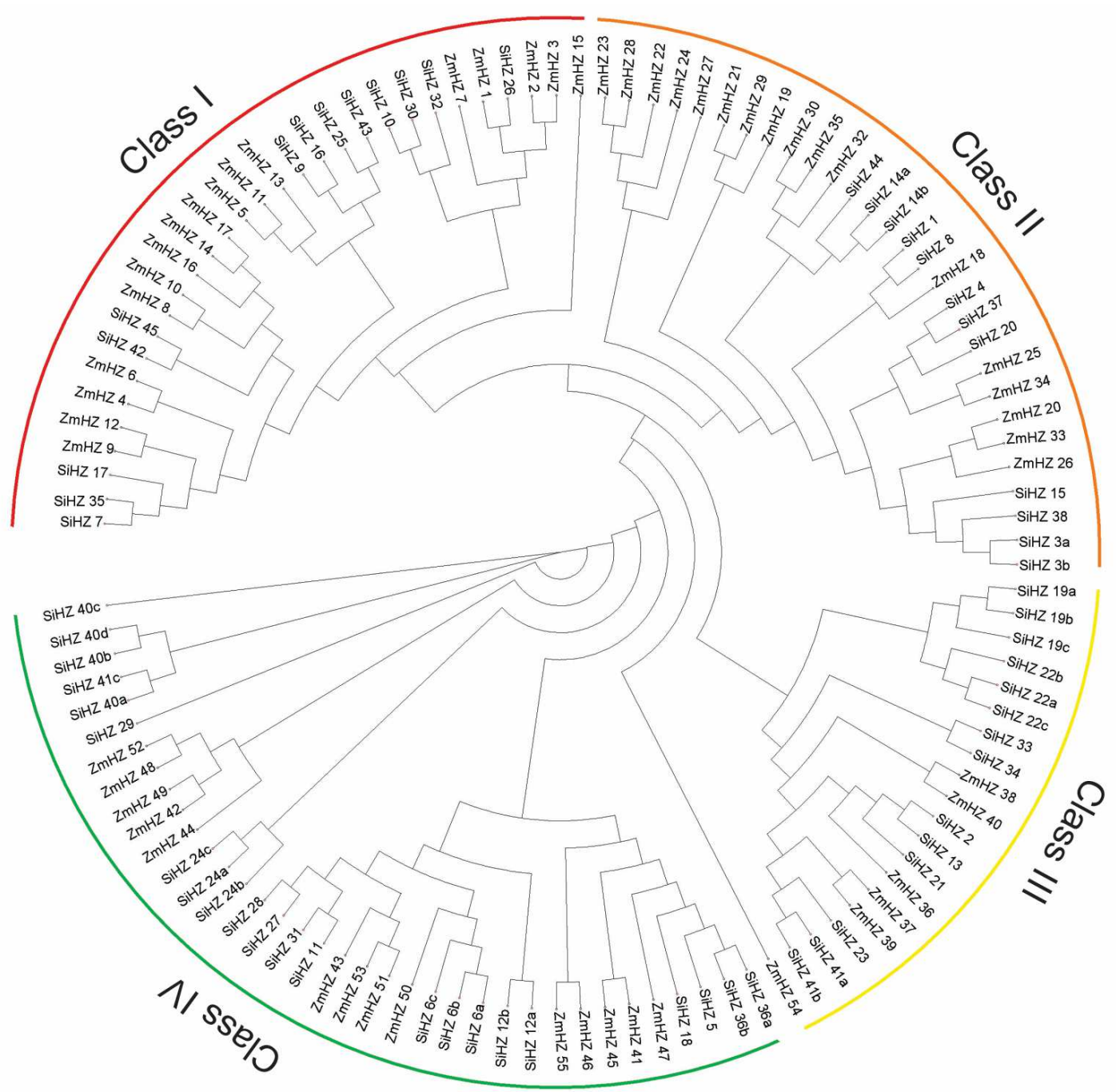

Fig. 1 Classification of HD-ZIP TF family in sesame - Maximum likelihood (ML) tree of 61 identified HD-ZIP transcription factors (TFs) from sesame. Full protein sequences of the HD-ZIP TF family in sesame were aligned by ClustalW and the ML tree was constructed by using MEGA (version X). Each class is labelled 
Fig. 2 Maximum likelihood (ML) tree of 13 identified HD-ZIP Class I transcription factors (TF) in sesame along with A. thaliana and Zea mays HD-ZIP Class I TFs. Full protein sequences of HD-ZIP Class I from sesame, A. thaliana and Zea mays were aligned by ClustalW, and the ML tree was constructed using MEGA (version X). Names of subgroups are indicated $(\alpha, \beta 2, \beta 1, \zeta, \delta, \varepsilon, \gamma$ and $\varphi)$ 
Class II

Class III

Class IV

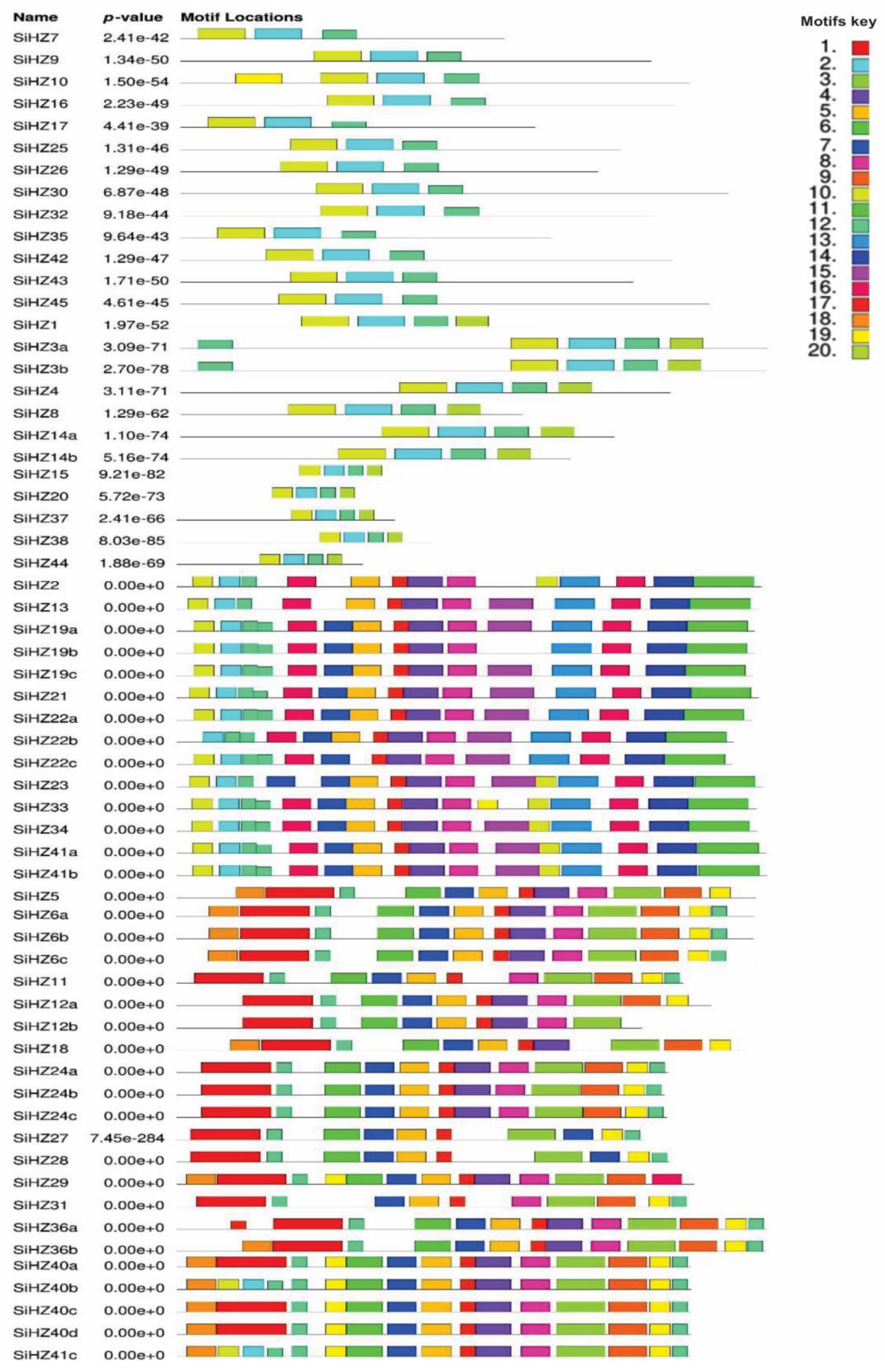

Fig. 3 The conserved domain analysis of HD-ZIP Classes (I-IV) protein sequences using MEME. Different colors represent the different conserved motifs. The motif sequences are presented in Supplementary Table 2 
(I)

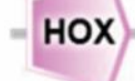

(II)

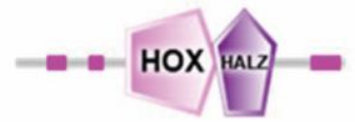

(III)

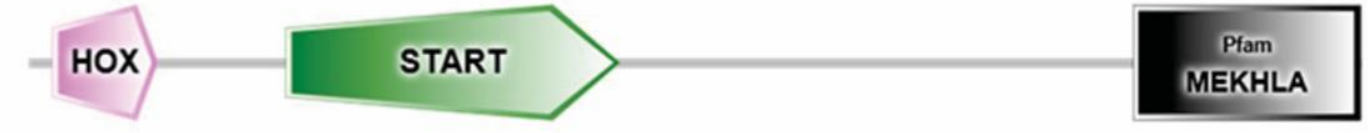

(IV)

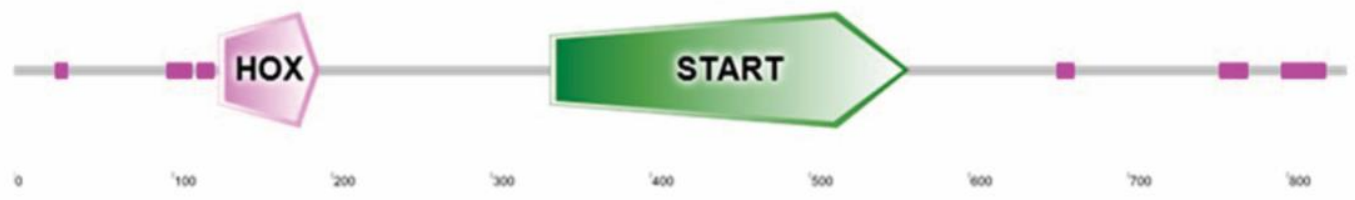

Fig. 4 Domain(s) architecture of HD-ZIP Class I-IV in sesame, predicted by SMART represents (I) SiHZ7 and other SiHZ members proteins of Class I; (II) SiHZ1 and other SiHZ members proteins of Class II; (III) SiHZ2 and other SiHZ members proteins of Class III; (IV) SiHZ5 and other SiHZ members proteins of Class IV 
Class 1

Class II

\begin{tabular}{|l|l|} 
SHZZ-10 \\
SHZZ-16 \\
SHAZ-17 \\
SHHZ-25 \\
SHZ-26 \\
SHZ.-30
\end{tabular}

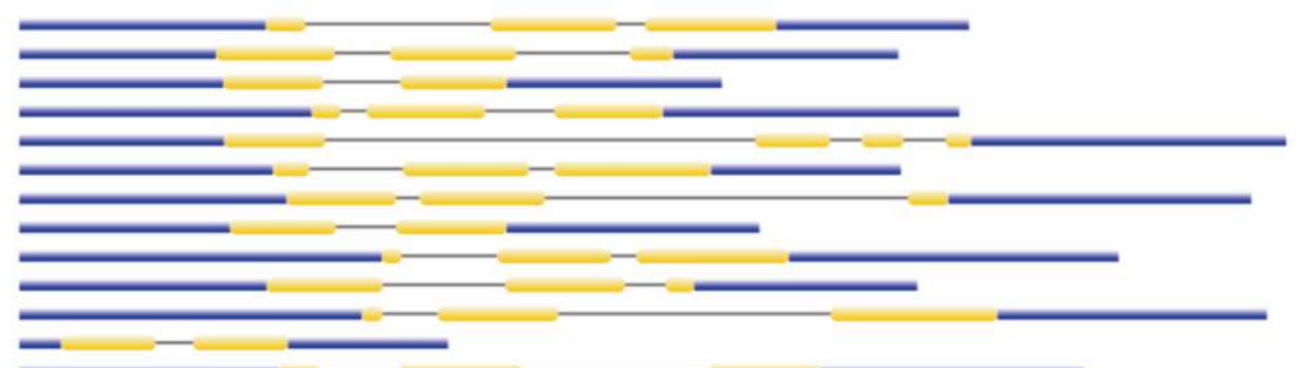

SiHz-.

SiHZ-1

SHZZ-14

SiHZ-140

SiHZ-15
SiHZ-20

SiHZ.37

SiHZ.-37
SiHZ. 38

SiHZ-3a

SiHZ-30

SiHZ-4

SiHZ.44

SiHZ-S

SiHZ-13

SHZZ-19a

Sill-196

SiHZ-19c

SiHZ.2

SHHZ-21

Class III SiHZ.22n

SAHZ-220

SiHZ.22c

SiHZ.23

SiHZ.-33

SiHZ-34

SiHZ-41n

SiHZ-4lb

SiHZ-11

SHZZ.12a

SiHZ-120

SAHZ-18

SiHZ.24a

SiHZ-240

SiHZ-24

SiHZ-27

Class IV

SiHZ 28
SiHZ. 29

SHZZ.360

SiHZ-360

SHZZ -40 a

SHZZ-400

SiHZ-40d

SiHZ-4le

SHHZ-S

SiHZ-6n

SiHZ-60

SiHZ-6c

Legend:

$-\mathrm{CDS}$

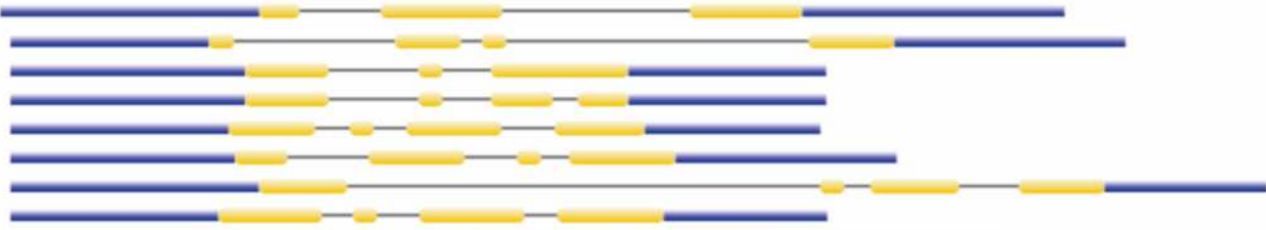

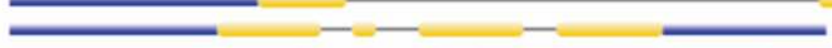
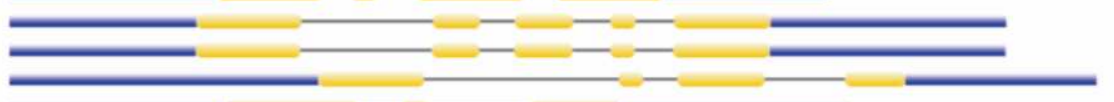

$\longrightarrow+-$

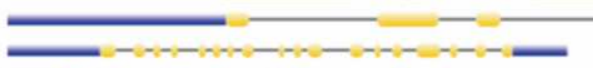

$m-m+m-m$

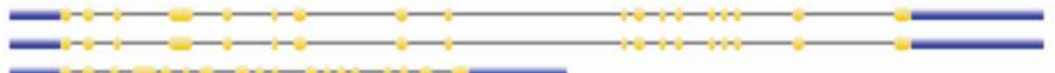

- $-1-\cdots-\cdots$

-

-

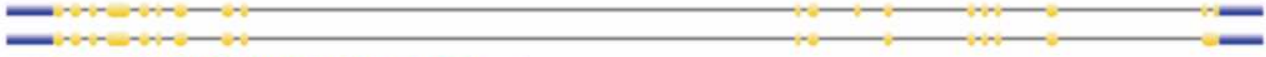

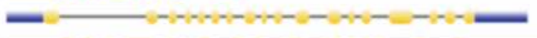

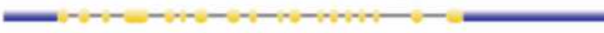

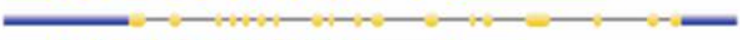

$[-1-$

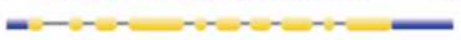

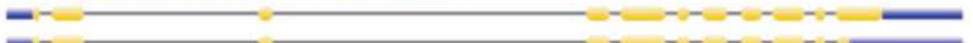

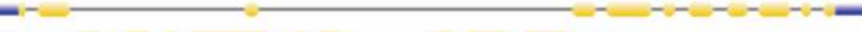

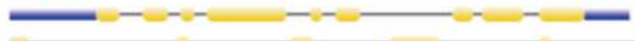

$+\cdots$

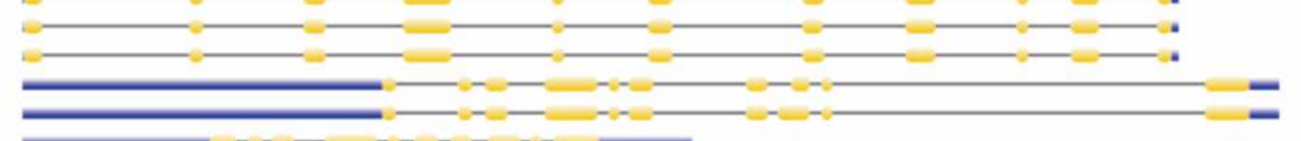

$\square+\ldots+-$

$\square-\ldots+-$

$\square-\ldots-\ldots-\ldots$

$\square-$

-

$\square-\ldots+-$

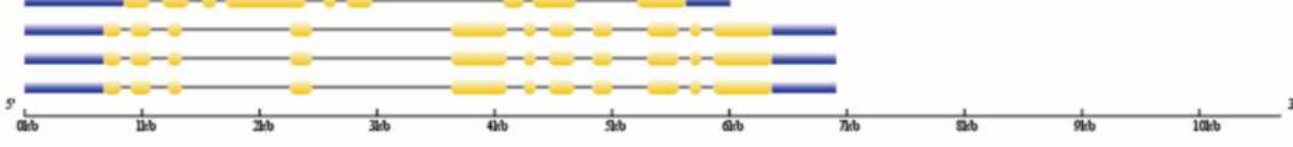

5

- upstrean/ downatream - Intron 


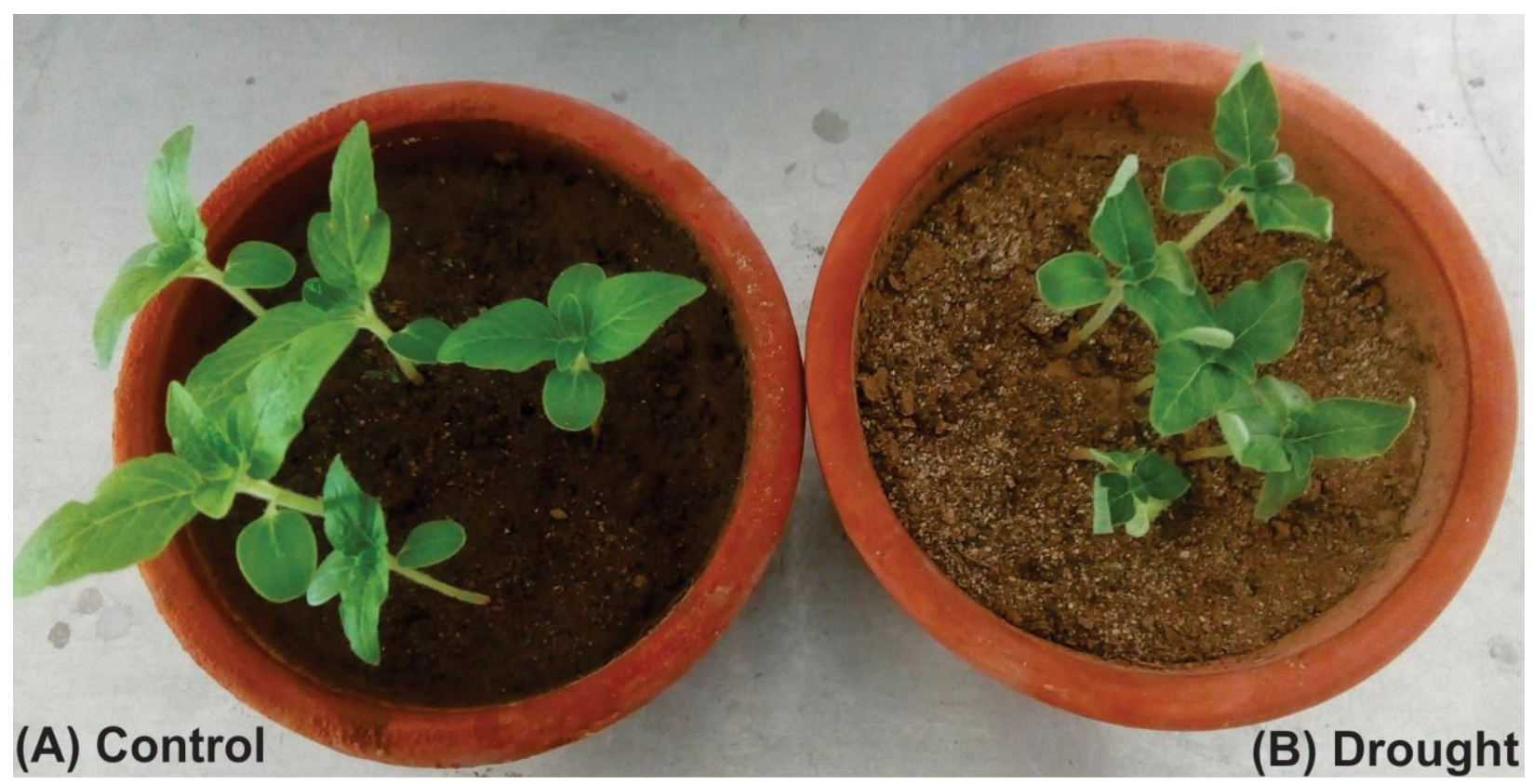

Fig. 6 Effect of drought stress on sesame early seedling growth. The seedlings were $>20$ days old, grown at $\pm 25{ }^{\circ} \mathrm{C}$ (A) Control (well-watered) seedlings (B) Drought stress was imposed to the seedlings by water withholding for 7 days and soil moisture content reached up to $\sim 16 \%$. The experiment was performed in three replicates 


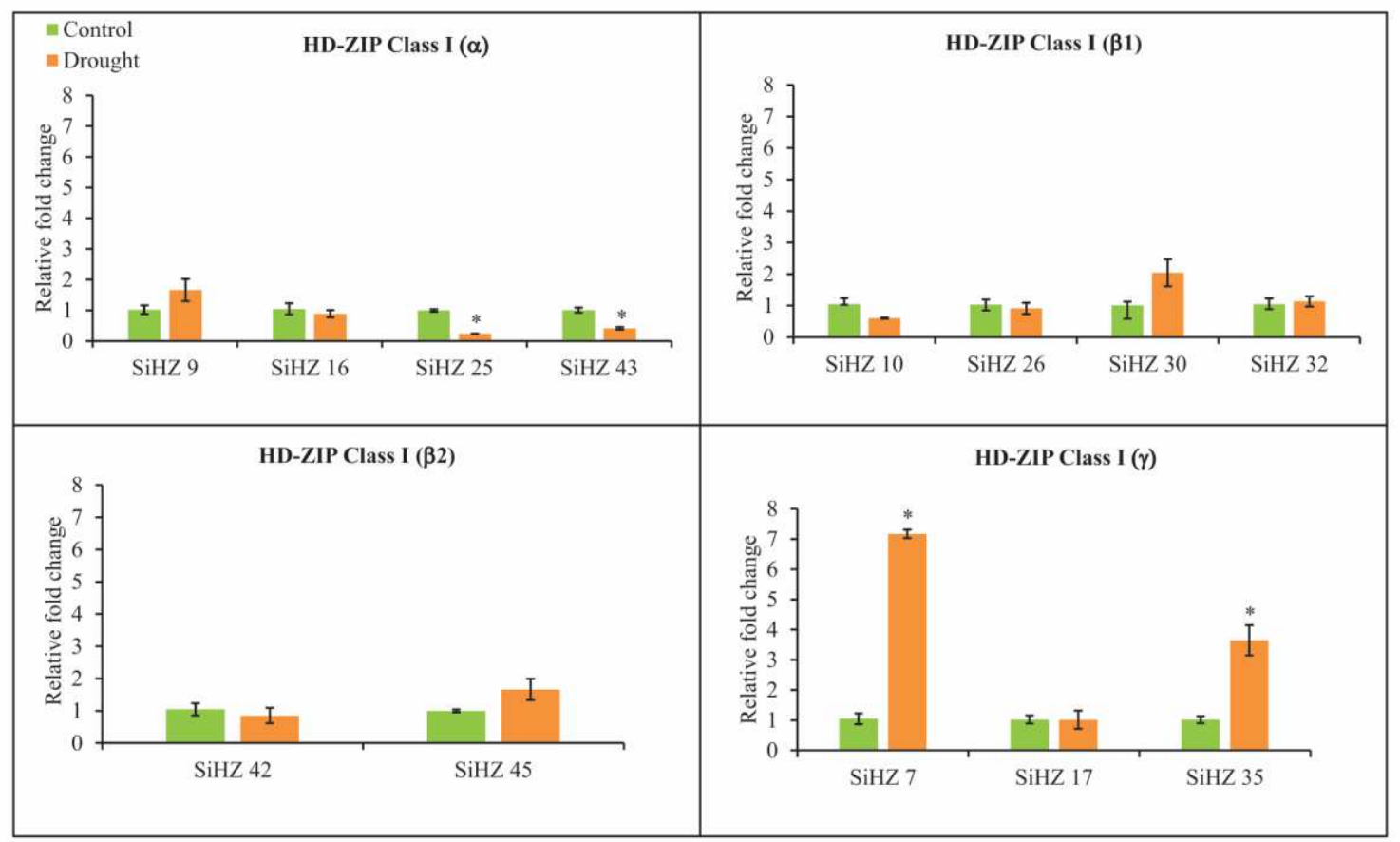

Fig. 7 Expression analysis of 13 identified HD-ZIP Class I genes by qPCR. The actin gene was used as reference gene. Subgroups: Values are means and capped lines are standard errors $(\mathrm{n}=3)$. *, Significant $(\mathrm{P}<0.05)$ change relative to the control 


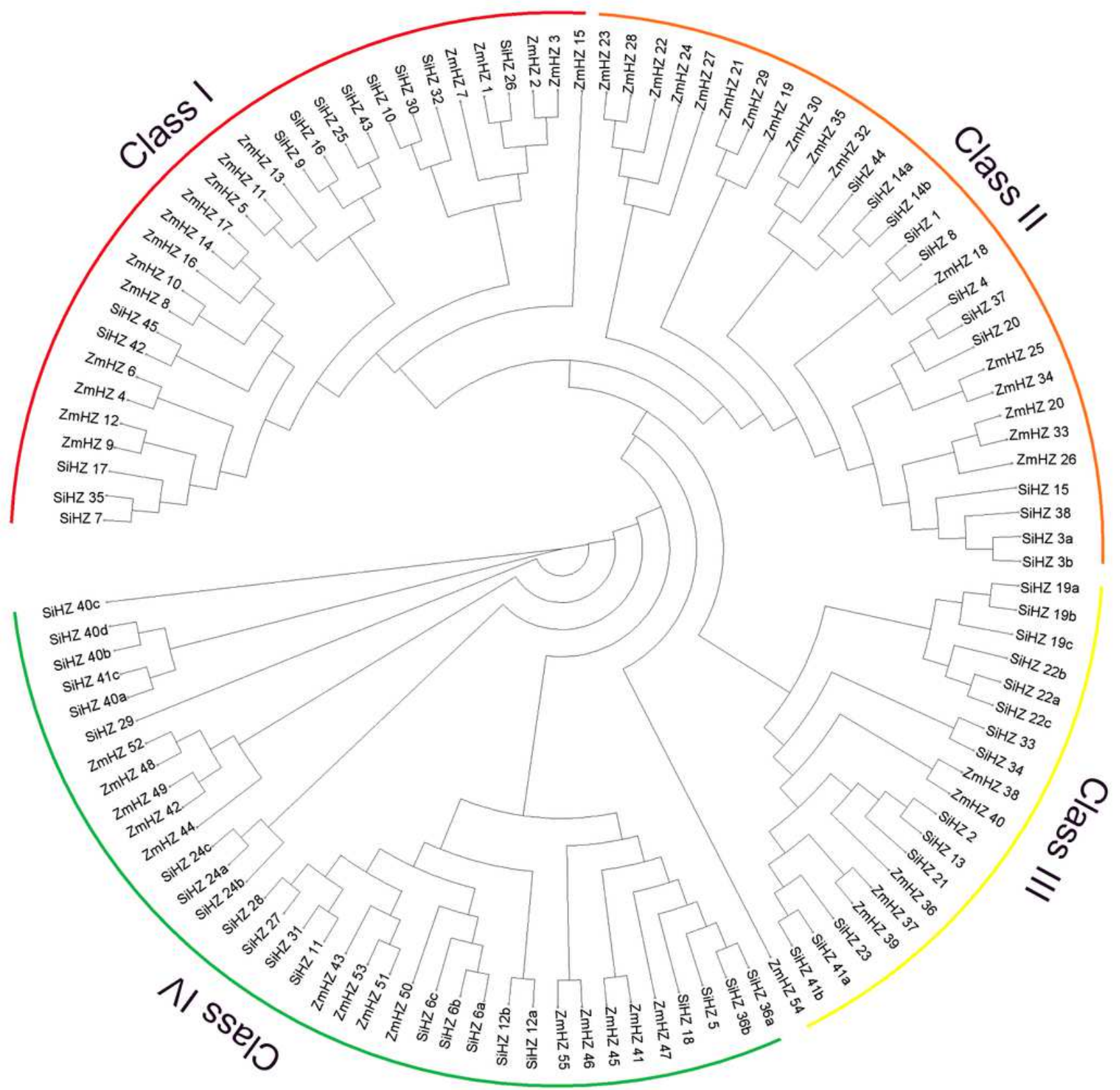

Figure 1

Classification of HD-ZIP TF family in sesame - Maximum likelihood (ML) tree of 61 identified HD-ZIP transcription factors (TFs) from sesame. Full protein sequences of the HD-ZIP TF family in sesame were aligned by ClustaIW and the ML tree was constructed by using MEGA (version $\mathrm{X}$ ). Each class is labelled 


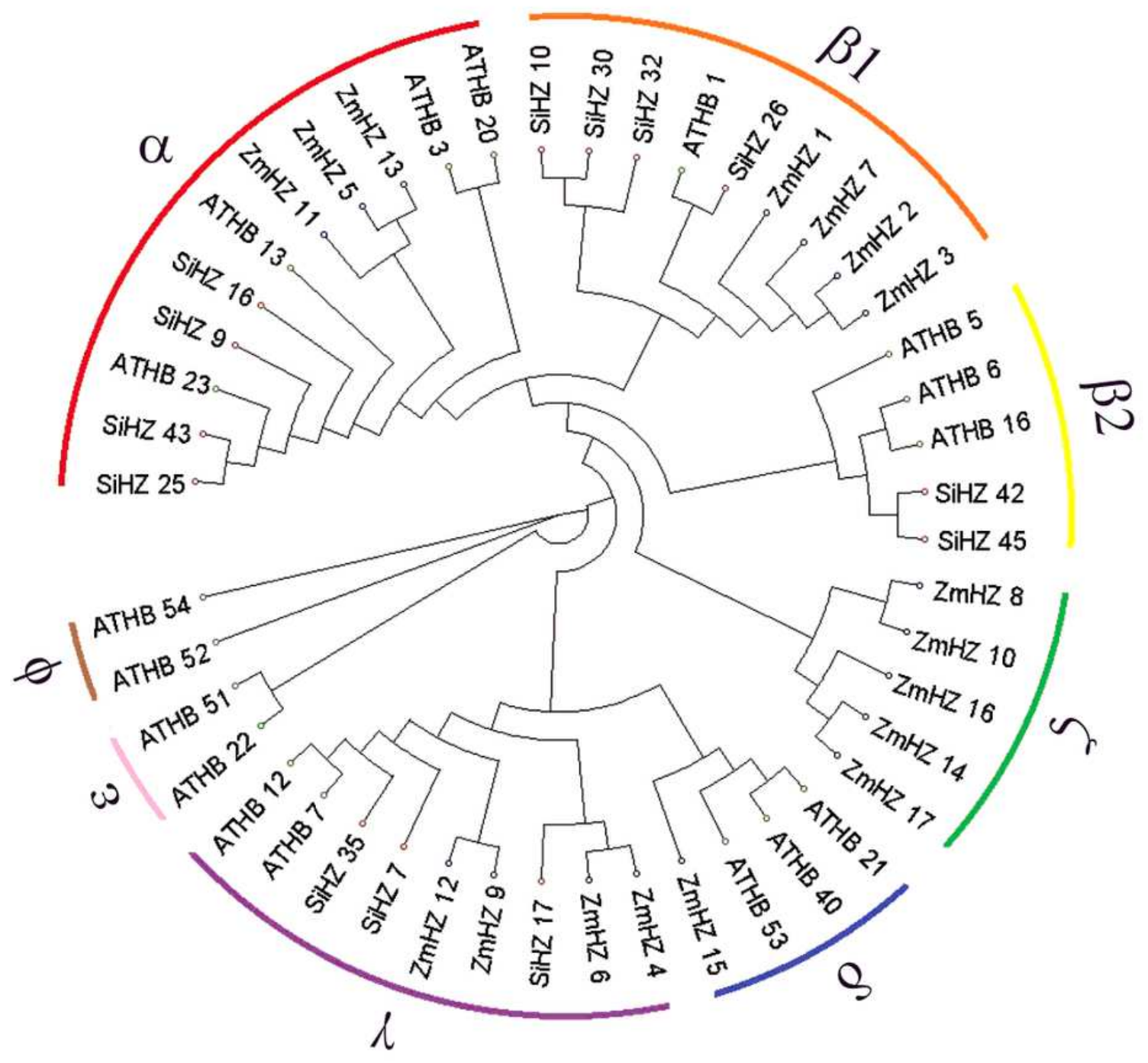

Figure 2

Maximum likelihood (ML) tree of 13 identified HD-ZIP Class I transcription factors (TF) in sesame along with A. thaliana and Zea mays HD-ZIP Class I TFs. Full protein sequences of HD-ZIP Class I from sesame, A. thaliana and Zea mays were aligned by ClustalW, and the ML tree was constructed using MEGA (version $X)$. Names of subgroups are indicated $(a, \beta 2, \beta 1, \zeta, \delta, \varepsilon, Y$ and $\varphi$ ) 


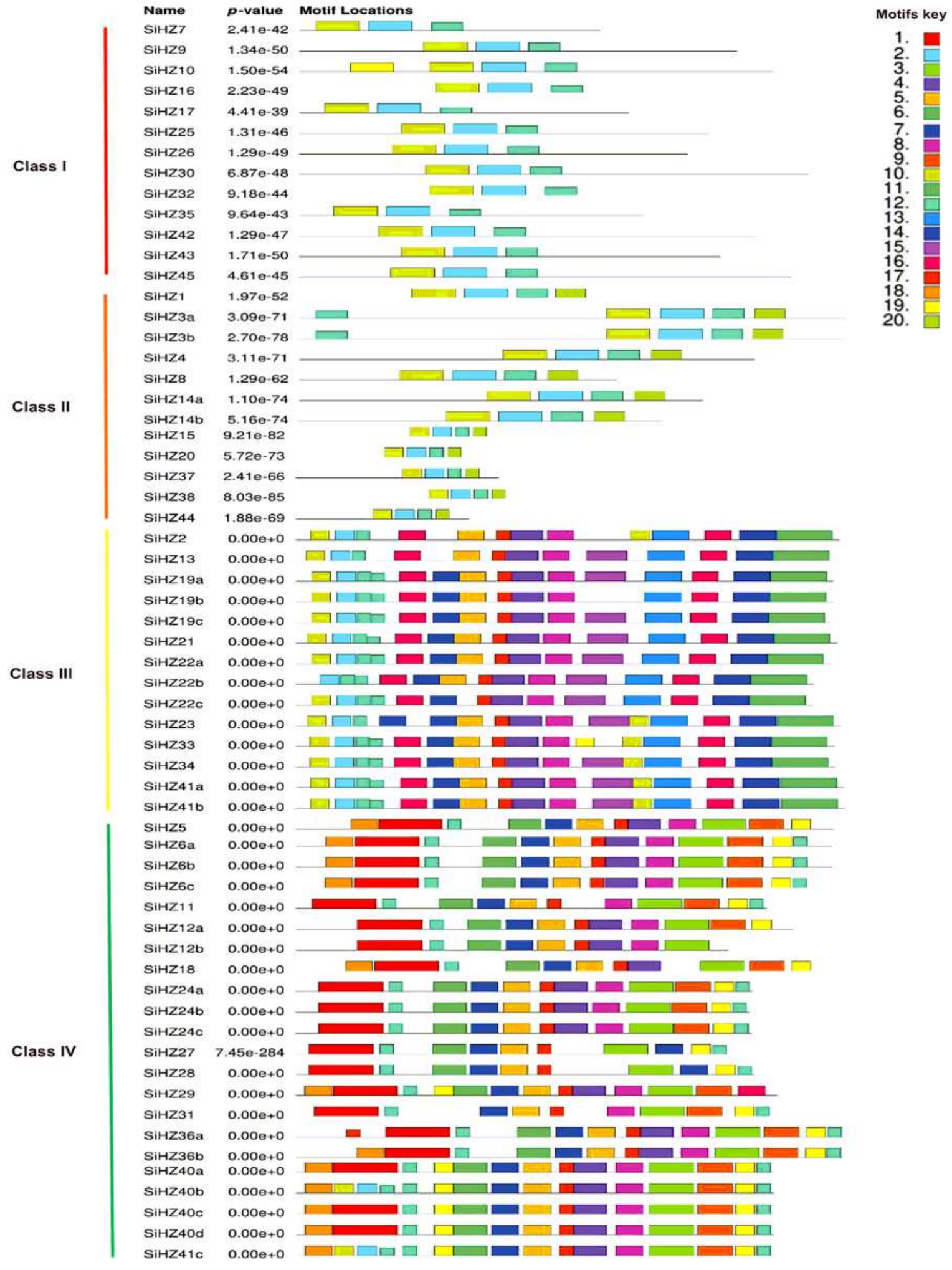

\section{Figure 3}

The conserved domain analysis of HD-ZIP Classes (I-IV) protein sequences using MEME. Different colors represent the different conserved motifs. The motif sequences are presented in Supplementary Table 2 


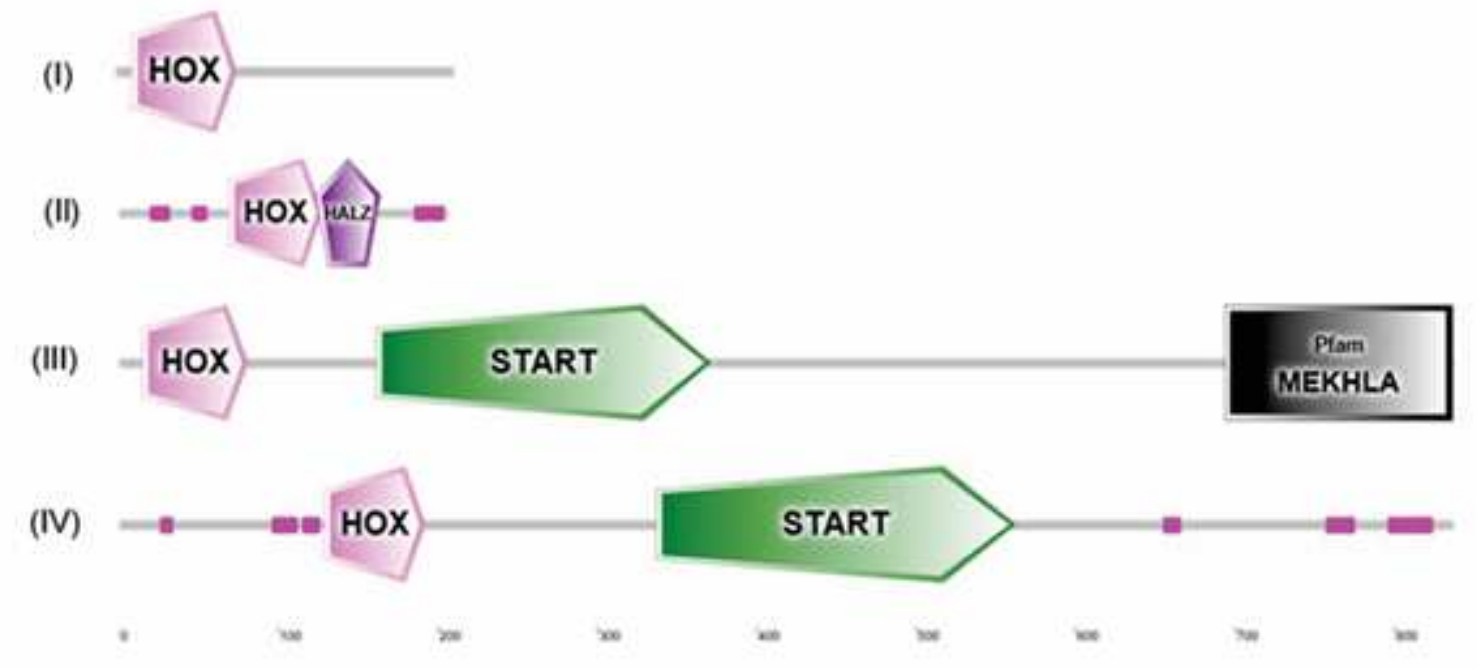

\section{Figure 4}

Domain(s) architecture of HD-ZIP Class I-IV in sesame, predicted by SMART represents (I) SiHZ7 and other $\mathrm{SiHZ}$ members proteins of Class I; (II) $\mathrm{SiHZ1}$ and other SiHZ members proteins of Class II; (III) $\mathrm{SiHZ2}$ and other SiHZ members proteins of Class III; (IV) SiHZ5 and other SiHZ members proteins of Class IV 


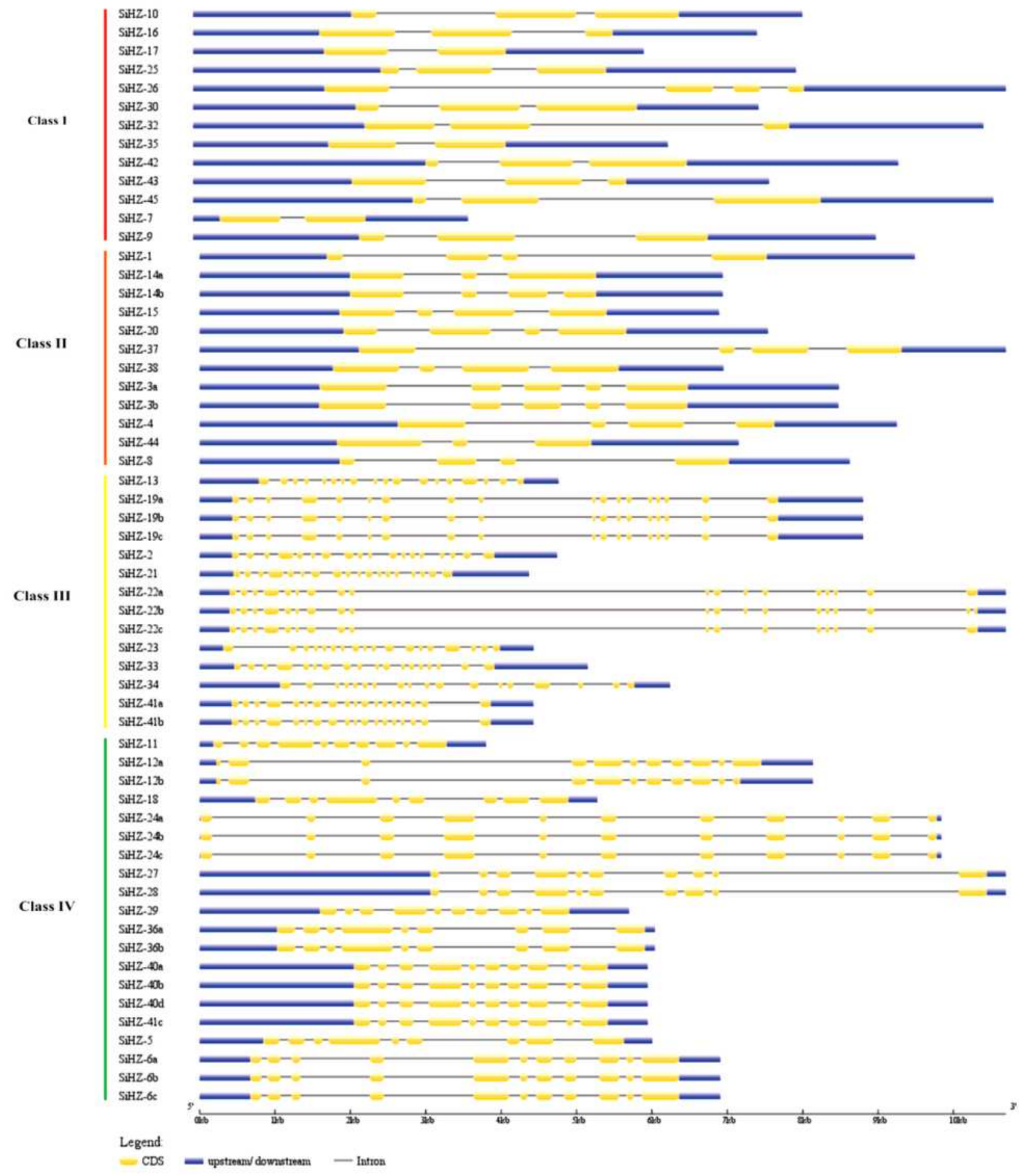

Figure 5

Gene structures of identified SiHD-ZIP Class I-IV by GSDS version 2.0 


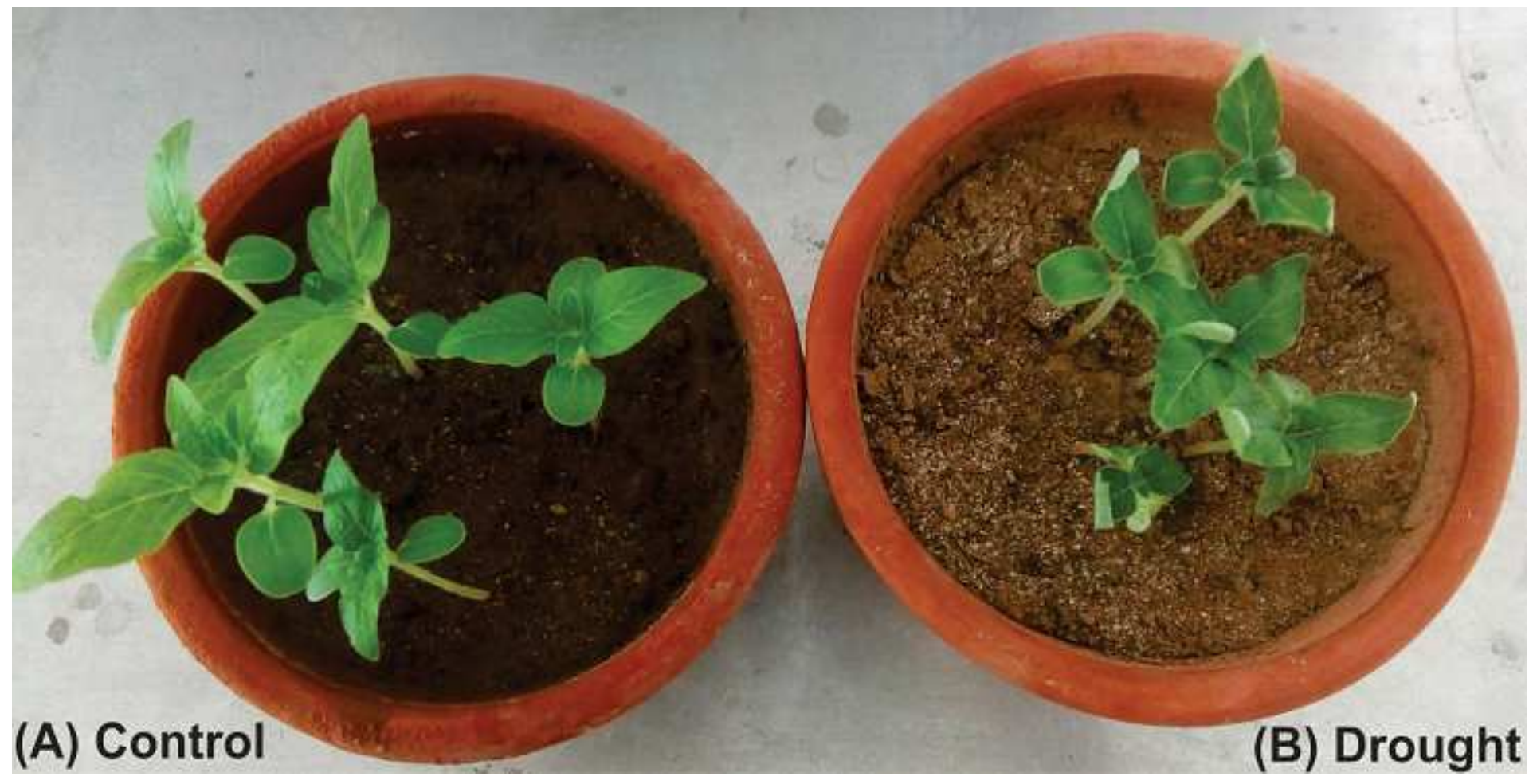

Figure 6

Effect of drought stress on sesame early seedling growth. The seedlings were $>20$ days old, grown at \pm 25 ${ }^{\circ} \mathrm{C}(\mathrm{A})$ Control (well-watered) seedlings (B) Drought stress was imposed to the seedlings by water withholding for 7 days and soil moisture content reached up to $\sim 16 \%$. The experiment was performed in three replicates 


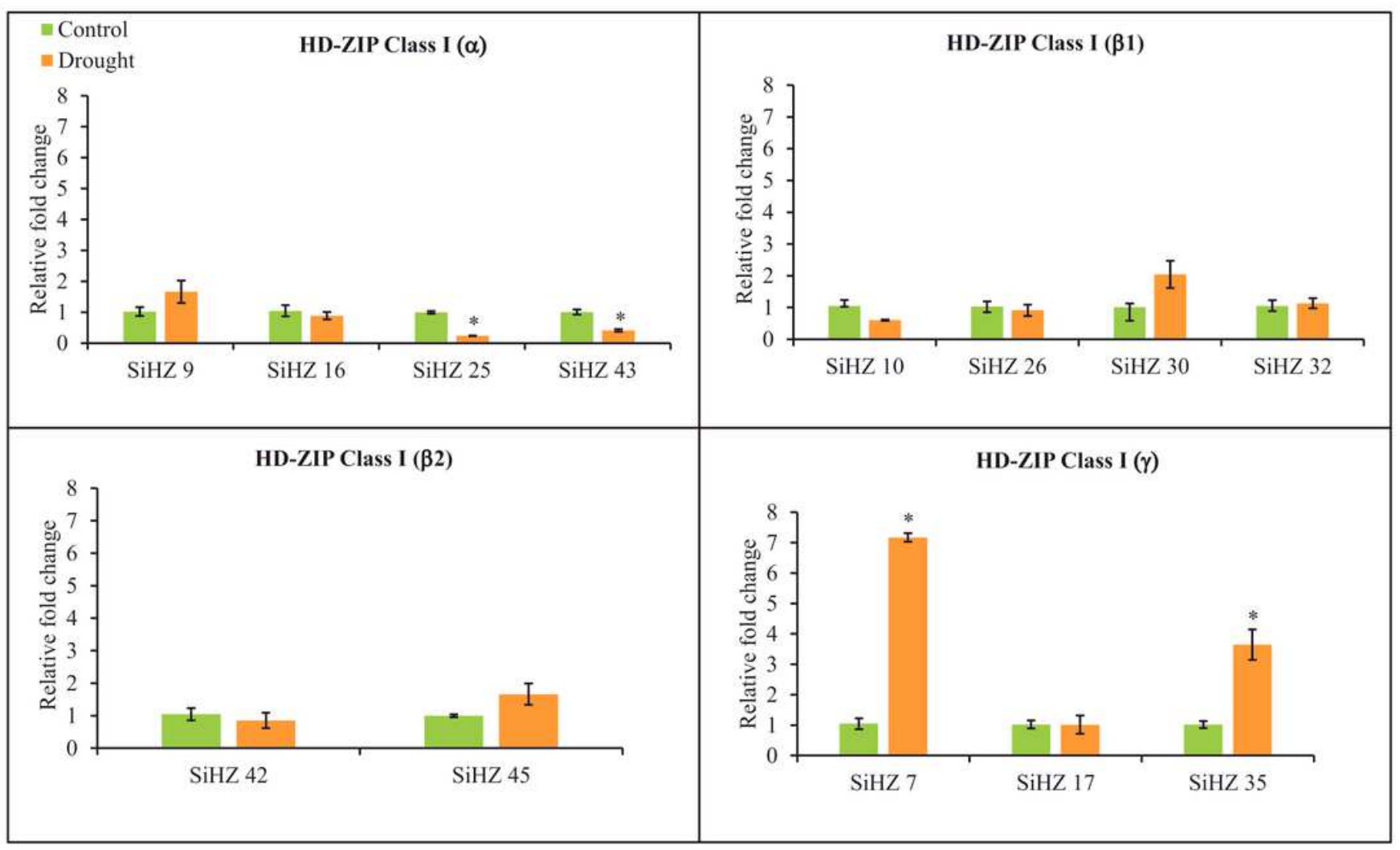

Figure 7

Expression analysis of 13 identified HD-ZIP Class I genes by qPCR. The actin gene was used as reference gene. Subgroups: Values are means and capped lines are standard errors $(n=3)$. ${ }^{*}$, Significant $(P<0.05)$ change relative to the control

\section{Supplementary Files}

This is a list of supplementary files associated with this preprint. Click to download.

- SupplementaryTables.docx 